\title{
Synthese einiger Erzmineralien und analoger Metallverbindungen durch Auflösen und Krystallisierenlassen derselben in geschmolzenen Metallen.
}

\author{
Von \\ Friedrich Roessler. \\ Mit 34 Figuren im Text.
}

Bei der künstlichen Darstellung von Mineralien auf trockenem Wege hat man häufig mit Erfolg einen Schmelzflufs angewendet, welcher, ohne Einflufs auf die chemische Zusammensetzung des Minerals, dasselbe bei höherer Temperatur gelöst enthält und beim langsamen Erkalten ihm Gelegenheit bietet, sich in Krystallen abzuscheiden. ${ }^{1}$

So hat Rose mittels des Phosphorsalzes $\mathrm{SiO}_{2}$, $\mathrm{TiO}_{2}, \mathrm{Fe}_{2} \mathrm{O}_{3}$ in krystallisierten Tridymit, resp. Anatas und Hämatit übergeführt. ${ }^{2}$ EBELmen hat Borsäure und Borax in mehreren Fällen als Schmelzmittel zu Krystallisationszwecken benutzt. ${ }^{3}$

Bourgeors wendet ein Gemenge gleicher Teile von Chlornatrium und Chlorkalium als Schmelzflufs an, um Karbonate, wie Calcit, Witherit, Strontianit, darin krystallisieren zu lassen. ${ }^{4}$

MARgotrer endlich hat den Schwefel als Schmelzflufs zur Darstellung krystallisierten Schwefelsilbers und Rotgüldigerzes benutzt. ${ }^{5}$ Bekannt ist die Darstellung krystallisierten Siliciums durch Auflösen desselben in Zink, des krystallisierten Bors durch Auflösen in Aluminium, sowie die Abscheidung des im geschmolzenen Eisen gelösten Kohlenstoffes als Graphit. Hierher gehört auch die von MoIssan ausgeführte Darstellung des Diamants durch Auskrystallisieren des Kohlenstoffes aus Eisen unter hohem Druck. ${ }^{6}$

Aufser den zuletzt genannten Fällen sind Metalle noch sehr selten als ,agent minéralisateur", wie französische Forscher derartige Substanzen nannten, ${ }^{7}$ benutzt worden.

1 Doecter, Chem. Miner. S. 120.

2 Ebendaselbst.

3 Meunier, Les méthodes de synthèse en minéralogie, S. 142.

Ebendaselbst S. 136.

- Compt. rend. 116, 218.

${ }^{5}$ Ebendaselbst S. 151.

${ }^{7}$ Meunier, Les méthodes de synthèse en minéralogie, S. 134. 
Im folgenden soll nun eine Reihe von Versuchen beschrieben werden, welche den Zweck verfolgen, Verbindungen verschiedener Schwermetalle, und zwar zunächst Sulfide und Selenide, dadurch krystallisiert zu erhalten, dafs man sie im Überschuls des betreffenden Metalles löst und beim Erkalten der geschmolzenen Masse sich wieder abscheiden läist.

Die erste Anregung zu diesen Untersuchungen gaben folgende Beobachtungen und Vorversuche, welche ich im Laboratorium der Scheideanstalt zu Frankfurt a. M. zu machen Gelegenheit hatte. Man hat dort mehrfach gefunden, dal's als unrein beanstandetes Scheidesilber, sowie einige Sorten Hüttensilber geringe Mengen Schwefel oder Selen enthalten, welche seine technische Brauchbarkeit beeinträchtigen, indem sie es leicht brüchig machen. Auch äufserlich sind solche schwefelhaltige Barren daran zu erkennen, dais ihre Oberflüche nicht spiegeiglatt ist, wie die des reinen Silbers, sondern krystallinische, reliefartig hervortretende Zeichnungen zeigt. Löst man solches Silber in heilser, konz. Salpetersäure, so lälst sich Schwefel und Selen in der Lösung als Schwefelsäure, resp. selenige Säure, nachweisen. So fand man in Mansfelder Silber $0.016 \% \mathrm{~S}$ und $0.0003 \% \mathrm{Se}$, in Silber von Pertusola in Oberitalien $0.0095 \%$ Se, in unreinem Scheidesilber, gewonnen aus Mansfelder Anodenschlämmen, $0.195 \%$ Se. Löst man aber solches Silber in kalter, verdünnter Salpetersäure (1.1 spez. Gew.), so hinterbleibt nicht etwa gelber Schwefel und rotes Selen, sondern ein körniger, metallisch glänzender, schwarzer Rückstand von Schwefelsilber, resp. Selensilber. Derselbe macht den Eindruck eines Krystallpulvers, zeigt aber unter dem Mikroskop keine erkennbaren Krystallformen. In der abfiltrierten Lösung lälst sich kein Schwefel oder Selen nachweisen. Auch bei der elektrolytischen Auflösung schwefelhaltigen Silbers hinterbleibt derselbe Rückstand bei Benutzung einer schwach salpetersauren Lauge, welche ebenfalls frei von Schwefelsäure bleibt.

Zur Bestätigung dieser Beobachtungen wurden folgende synthetische Versuche gemacht. $50 \mathrm{~g}$ feiner Silberkryställchen wurden mit $0.5 \mathrm{~g}$ Schwefel gemischt, im Tiegel zusammengeschmolzen und in Wasser ausgegossen. $20 \mathrm{~g}$ wurden in heilser Salpetersäure gelöst, der Schwefelgehalt als $\mathrm{BaSO}_{4}$ bestimmt, und daraus der Gehalt an Schwefelsilber berechnet. In einer zweiten Menge von $20 \mathrm{~g}$, die, um schneller gelöst zu werden, platt gewalzt waren, wurde das Schwefelsilber direkt als der Rückstand bestimmt, der beim Auflösen in verdünnter, kalter, Salpetersäure (1.1 spez. Gew.) blieb. In 
$20 \mathrm{~g}$ fanden sich $1.3110 \mathrm{~g} \mathrm{BaSO}_{4}=0.97 \% \mathrm{~S}$. Daraus berechnet sich der Schwefelsilbergehalt auf $7.50 \%$. Gefunden wurden $7.18 \%$, nämlich $0.6690 \mathrm{~g}$ Rückstand in $20 \mathrm{~g}$.

Dals die hier angegebenen Werte für die wirklich gefundene und die aus dem Schwefelgehalt berechnete Menge Schwefelsilber nicht genauer übereinstimmen, hat seinen Grund mit darin, dals, wie sich bei späteren Versuchen zeigte, das Schwefelsilber nicht in der ganzen Masse gleichmälsig verteilt ist.

Ein analoger Versuch wurde mit $25 \mathrm{~g}$ Silber und $0.3 \mathrm{~g}$ Selen angestellt. Der Selengehalt des Silbers läist sich in diesem Falle sehr bequem als selenigsaures Silber bestimmen, da dieses in Wasser so gut wie unlöslich ist. Man dampft zu diesem $Z_{w e c k e}$ die salpetersaure Lösung zur Trockne, nimmt mit kaltem Wasser auf und wägt den filtrierten Rückstand auf einem gewogenen und bei $100^{\circ}$ getrockneten Filter.

Es fanden sich in $8.69 \mathrm{~g}$, die in heifser Salpetersäure gelöst wurden, $0.3945 \mathrm{~g} \mathrm{Ag}_{2} \mathrm{SeO}_{3}=1.03 \%$ Se. Daraus berechnet sich der Gehalt an Selensilber auf $3.88 \%$. Gefunden wurden $4.06 \%$, nämlich in $9.48 \mathrm{~g} 0.3845 \mathrm{~g}$ Rückstand beim Auflösen in kalter, verdünnter Salpetersäure.

In beiden Fällen liefs sich beim Auflösen in kalter verdünnter Säure in derselben kein Schwefel oder Selen nachweisen. Die Versuche zeigen also, dals aller im Silber enthaltene Schwefel, ebenso wie das Selen, sich beim Erkalten in der Masse des Silbers als Schwefelsilber, resp. Selensilber, abscheidet.

Es schien nun naheliegend, die hier beobachtete Abscheidung eines Schwefelmetalles in der Masse des geschmolzenen Metalles zur Synthese von krystallisierten Schwefel- resp. Selenverbindungen zu benutzen. Um zunächst die Vorgänge bei der Lösung und Ausscheidung der Verbindung im geschmolzenen Metall klar zu legen, und die Frage zu studieren, wieweit dabei überhaupt die Bildung von Krystallen möglich ist, wurde eine Anzahl Schmelzungen von Silber mit wechselnden Mengen von Schwefel und Selen gemacht.

Die im folgenden zu beschreibenden Schmelzversuche wurden fast alle in dem Rössuerschen Gasöfchen angestellt. Das Prinzip desselben ist, wie bekannt, einmal die erzeugte Wärme möglichst an dem Punkt, an welchem sie gebraucht wird, d. h. um den Schmelztiegel, zu konzentrieren und möglichst wenig Wärme nach aufsen dringen zu lassen, zweitens die zum Verbrennen des Leuchtgases nötige Luft durch die abziehenden Verbrennungsgase vorZ. anorg. Chem, 1X, 
zuwärmen. Bei einiger Übung kann man leicht durch Änderung der Luft- und Gaszufulr die Temperatur in dem Ofen regulieren. Man kann in demselben in ca. 15 Minuten Goldschmelzhitze (ca. $1050^{9}$ ) erzielen und bei längerem Brennen eine Legierung ron $85 \%$ $\mathrm{Au}$ und $15 \% \mathrm{Pt}$ schmelzen (ca. 1200\%). Wendet man geprefste Luft an, so kann man leicht die Schmelztemperatur des Palladiums (ca. $1400^{\circ}$ ) erreichen. Die erzeugten Temperaturen können durch das Schmelzen ron Legierungen ron bekanntem Schmelzpunkt kontrolliert und dazu Legierungen ron Gold und Platin nach der bekannten Skala von Prinsep rerwendet werden. Ist der Ofen einmal heifs, so hält er die Hitze lange fest, ein Umstand, der sehr wesentlich ist, wemn es sich darum handelt, geschmolzene Metalle langsam erkalten zu lassen.

Bei einigen Schmelzversuchen wurde auch mit Vorteil ein Muffelofen angewendet, wie er zum Abtreiben der Gold- und Silberproben benutzt wird. Da die Temperatur in dem vorderen Teil der Muffel keine Silberschmelzhitze erreicht, am hinteren Ende derselben aber Goldschmelzhitze übersteigt, so bietet dieser Ofen den Vorteil, zu gleicher Zeit über eine Reihe verschiedener Hitzegrade verfügen zu können. Auch gestattet derselbe leichter, die Vorgänge beim Schmelzen zu beobachten. Zur Erzielung sehr hoher Temperaturen wurde ein Koksofen mit Gebläse nach Deviche benutzt. Schmelzungen mit gröIseren Mengen Metall wurden in gewöhnlichen Silberschmelzöfen mit Koksfeuer ausgeführt.

\section{Schwefel- und Selenverbindungen.}

Schwefelsilber und Selensilber im Silber.

Es wurden in dem oben beschriebenen Gasofen je $20 \mathrm{~g}$ Feinsilber in einem kleinen Tiegel von feuerfestem Thon unter Borax eingeschmolzen und in dasselbe wechselnde Mengen von Schwefelsilber $(2-30 \%)$ eingetragen. Das Schwefelsilber war teils auf nassem Wege, teils durch Zusammenschmelzen von elektrolytisch ausgefällten Silberkryställchen mit überschüssigem Schwefel unter Borax erhalten. Es sank in dem Borax sofort unter, so dals ein Verbrennen von Schwefel ziemlich ausgeschlossen war. Der Tiegel wurde eine halbe Stunde in heller Rotglut gehalten und während etwa zwei Stunden im Ofen langsam erkalten lassen. Der so erhaltene König wurde durchsägt. Mit zunehmendem Schwefelsilbergehalt wurde der Bruch rauher und die Farbe desselben, wie die der Schnittfäche, dunkler. 
Betrug die eingetragene Menge des Schwefelsilbers mehr als etwa $20 \%$, so erschien der Durchschnitt nicht mehr homogen, sondern es zeigten sich in demselben mehrere dunkle Flecke und obenauf eine dunkle Schicht. Beim Auflösen der Könige in verdünnter, kalter Salpetersäure (1.1 spez. Gew.) hinterblieben regelmä[sig glänzende, schwarze Körperchen, die jedoch niemals krystallisiert waren, sondern unter dem Mikroskop unregelmälsige, abgerundete, erstarrten Tropfen ähnliche Formen zeigten (Fig. 1).

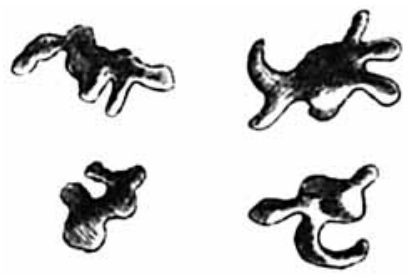

Fig. 1.

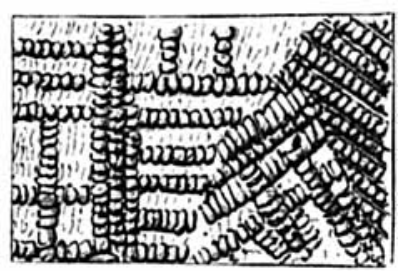

Fig. 2.

Obwohl also die Darstellung von Krystallen auf diese Weise nicht zu gelingen schien, wurden einige gröfsere Schmelzungen gemacht, um zunächst zu versuchen, Einblick in die Vorgänge beim Erstarren der Metallösung zu gewinnen. Eine Lösung von Schwefelsilber in Silber erwies sich aus folgenden Gründen zu solchen Versuchen als sehr geeignet. Erstens lassen sich wegen des Farbenunterschiedes zwischen dem weifsen Silber und dem schwarzen Schwefelsilber die Erscheinungen auf der Schnittläche gut beobachten. Ferner wird Silber von kalter, verdünnter Salpetersäure (1.1 spez. Gew.) leicht gelöst, während Schwefelsilber nicht angegriffen wird. Drittens liegt der Schmelzpunkt des Silbers (ca. 950) ziemlich entfernt von dem des Schwefelsilbers (ca. 850\%).

Es wurden nun folgende drei Schmelzungen gemacht.

Sehmelzung I. $400 \mathrm{~g}$ elektrolytisch ausgefällte Feinsilberkryställchen wurden mit $1 \mathrm{~g}$ Schwefel (entsprechend ca. $2 \%$ Schwefelsilber) innig gemischt, unter Borax eingeschmolzen und langsam erkalten lassen.

Schmelzung II. $300 \mathrm{~g}$ Silber mit $20 \mathrm{~g}$ Schwefel (entsprechend ca. $50 \%$ Schwefelsilber) wurden ebenso eingeschmolzen und langsam erkalten lassen.

Schmelzung III. $300 \mathrm{~g}$ Silber mit $20 \mathrm{~g}$ Schwefel (entsprechend ea. 50\% Schwefelsilber) wurden durch Ausgielsen in eine kalte eiserne Form rasch erkalten lassen.

Die erhaltenen Könige wurden durchschnitten, die Schnittflächen poliert und mit konz. Salpetersäure angeätzt. Sie boten folgende charakteristische Erscheinungen. 
König I, ca. $2 \%$ Schwefelsilber enthaltend; zeigte auf seiner Oberfläche die krystallinischen Zeichnungen, wie sie an unreinem Silber beobachtet werden, in hervorragendem Mafse (Fig. 2). Die Schnittfläche erschien dem unbewaffneten Auge homogen und silberweils. Mit dem Mikroskop liefsen sich aber zu unregelmälsigen Linien angeordnete dunkle Punkte erkennen, die zwischen rein weifsen Flächen ein feines Netzwerk bildeten (Fig. 3).

König II, ca $50 \%$ Schwefelsilber enthaltend, zeigte zwei scharf abgegrenzte Teile, oben eine schwarze Schicht, etwa ein Drittel der ganzen Masse, und darunter eine helle Schicht, in welcher mehrere schwarze Einschliefsungen von unregelmälsiger Gestalt safsen (Fig. 4). Unter dem Mikroskop zeigte sich, dafs der ganze helle Teil des Königs mit schwarzen Körperchen durchsetzt war, die aber weder eine bestimmte Gestalt, noch eine bestimmte Anordnung erkennen liefsen. Ebenso safsen in der oberen dunklen Schicht einige

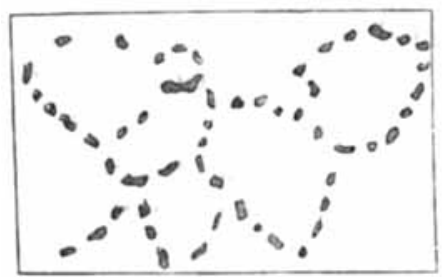

Fig. 3.

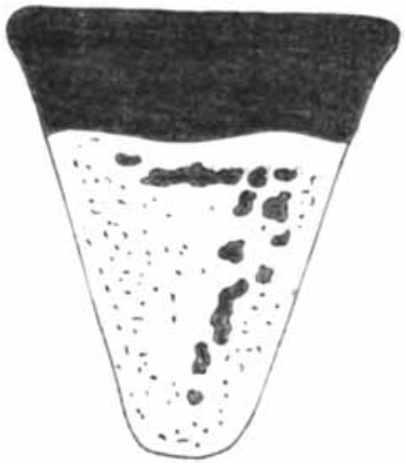

Fig. 4.

weil'se kleine Körperchen, von welchen jedoch einige unter dem Mikroskop eine federartige Anordnung ohne scharfe Formen zeigten (Fig. 5 und 6). Die gröIseren Einschlüsse, mit verdünnter Salpetersäure herausgelöst, besafsen keine Krystallformen, doch liefsen sich an einem derselben mit der Lupe krystallinische Zeichnungen beobachten, die sich wie ein Abdruck der Krystallbildungen darstellten, die langsam erstarrtes Silber auf seiner Oberfläche zeigt.

Ein scheinbar homogenes, aus der Mitte des Königs ausgeschnittenes Stück wurde in verdünnter kalter Salpetersäure gelöst. In der Lösung lieís sich kein Schwefel nachweisen. Es hinterliefsen dabei $2.6880 \mathrm{~g} 0.4560 \mathrm{~g}=17.00 \%$ schwarzen Rückstand, der wieder 
die obenerwähnten Formen, aber keine Krystalle zeigte. Derselbe bestand aus reinem Schwefelsilber. $0.8943 \mathrm{~g}$ Substanz ergaben $1.0282 \mathrm{~g} \mathrm{AgCl}$ und $0.8138 \mathrm{~g} \mathrm{BaSO}_{4}$.

\begin{tabular}{|c|c|}
\hline Gefunden: & Berechnet: \\
\hline $87.11 \% \mathrm{Ag}$ & $87.06 \% \mathrm{Ag}$ \\
\hline $12.52, \mathrm{~S}$ & $12.94, \mathrm{~S}$ \\
\hline $99.63 \%$ & $00 \%$ \\
\hline
\end{tabular}

Ebenso ergab sich die obere dunkle Schicht, sowie auch die grölseren Einschlüsse als Schwefelsilber. $1.4605 \mathrm{~g}$ der oberen Schicht ergaben $1.6930 \mathrm{~g} \mathrm{AgCl}=87.25 \%$ Ag. $0.1527 \mathrm{~g}$ der Einschlüsse ergaben $0,1745 \mathrm{~g} \mathrm{AgCl}=86.00 \% \mathrm{Ag}$.

Der rasch erkaltete König III mit ca. 50\% Schwefelsilber zeigte ebenfalls zwei getrennte Schichten. Jedoch fehlten hier völlig die gröIseren Einschlüsse in der hellen Schicht. Unter dem Mikroskop erwies sich aber der ganze belle Teil mit kleinen, unregelmälsig verteilten schwarzen Körperchen durchsetzt, welche für das unbe-

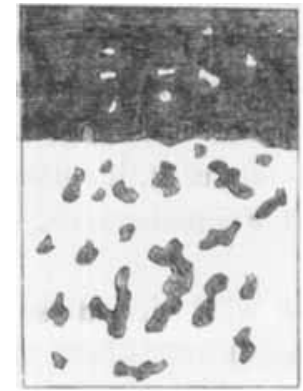

Fig. ธิ.

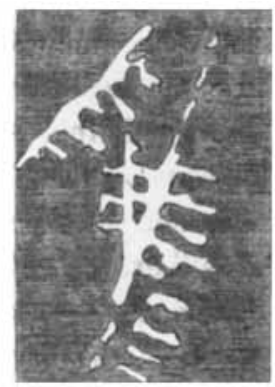

Fig. 6.

waffnete Auge eine gleichmälsige Graufärbung hervorriefen. Ein homogen aussehendes Stück aus der Mitte des Königs wurde in kalter verdünnter Salpetersäure $(1.1 \mathrm{spez.}$ Gew.) gelöst. In der Lösung liels sich kein Schwefel nachweisen. Dabei hinterlielsen $1.8885 \mathrm{~g}$ Material $0.3485 \mathrm{~g}=18.46 \%$ Rückstand. Derselbe bildete ein schwarzes, glänzendes Pulver, welches unter dem Mikroskop wieder die schon beschriebenen abgerundeten Formen zeigte, nur dals die einzelnen Körperchen kleiner waren als bei König II. Sie erwiesen sich ebenfalls als $\mathrm{Ag}_{2} \mathrm{~S}$. $0.5525 \mathrm{~g}$ Substanz ergaben $0.6356 \mathrm{~g}$ $\mathrm{AgCl}$ und $0.5060 \mathrm{~g} \mathrm{BaSO}_{4}$. 


$\begin{array}{cc}\text { Gefunden: } & \text { Berechnet: } \\ 87.12 \% \mathrm{Ag} & 87.06 \% \mathrm{Ag} \\ 12.56 \% \mathrm{~S} & 12.94 \% \mathrm{~S} \\ 99.68 \% & 100.00 \%\end{array}$

Die hier gemachten Beobachtungen kann man sich an der Hand der Theorieen über Gefrieren von Lösungen, insbesondere über Erstarren von Legierungen, wie sie in OsTwaLds Lehrbuch ${ }^{1}$ entwickelt sind, folgendermalsen erklären. Das geschmolzene Silber vermag je nach seiner 'Temperatur eine gewisse Menge Schwefelsilber zu lösen, und zwar in der Nähe seines Schmelzpunktes 18-19\%. Ein Überschufs von Schwefelsilber setzt sich oben auf und vermag seinerseits auch wieler etwas Silber zu lïsen, wie die Abscheidung von weil'sen Silberteilchen in der Schwefelsilberschicht zeigt. Man hat dann einen der Lösung zweier nicht vollkommen mischbarer Flüssigkeiten, etwa wie Äther und Wasser, analogen Fall. Wäre keine wirkliche Lösung, sondern nur ein mechanisches Gemenge vorhanden, so wäre nicht einzusehen, warum nur ein Teil des spezifisch leichteren Schwefelsilbers sich oben aufsetzt. Zudem spricht für die Annahme einer Lösung die Erniedrigung des Erstarrungspunktes des Silbers durch einen Gehalt an Schwefelsilber. Es wurde nämlich durch Schmelzversuche im Muffelofen konstatiert, dals Silber mit $15 \%$ Schwefelsilber später wie Feinsilber und ungefähr gleichzeitig mit einer Legierung von $950 \mathrm{Ag}+50 \mathrm{Cu}$, d. h. etwa bei $900^{\prime \prime}$, erstarrt, während der Schmelzpunkt des Feinsilbers ca. 950", der des Schwefelsilbers ca. $850^{\circ}$ ist.

Beim langsamen Erkalten der Masse wird sich diese Lösungsfäligkeit des Silbers verringern und sich Schwefelsilber abscheiden, welches sich zuerst noch mit der oberen Schicht vereinigt. Es wird dann der Punkt kommen, wo sich in der geschmolzenen Masse Silberkrystalle zu bilden beginnen, d. J. das Silber wird gefrieren und das in dernselben gelöste Schwefelsilber ausfrieren. Die zwischen den Silberkrystallen befindliche Lösung ron Schwefelsilber in Silber bleibt stets gesättigt. Das aus ibr ausfrierende Schwefelsilber wird zunächst zusammentreten können und nach oben zu dringen suchen, zum Teil aber durch die festen Silberkrystalle daran grehindert und von ihnen eingeschlossen werden, welcher Vorgang zur Bildung der beobachteten grölseren Einschlüsse führt. Bei weiterem Sinken der Temperatux wird daun der Gefrierpunkt der gesättigten Lösung er-

1 Ostwato, Lehbuch der allgem. Chemie. (2. Aufl.) 1, 101S fï. 
reicht werlen und diese dam die letzten Reste des gelösten Schwefelsilbers treigeben.

Tritt die Erkaltung sebr rasch ein, so wird sich das gelöste Schwefelsilber in sehr kurzer Zeit völlig ausscheiden. Es wird aber zwischen den schon erstarrten Silberkrystallen nicht mehr nach oben dringen oder zu gröfseren Partieen zusammentreten können, sondern ebenfalls sofort erstarren und so in kleinen Partikelchen in der ganzen Masse gleichmälsig verteilt bleiben. Daher wird die Menge des aus dem rasch erkalteten König III ausgelösten Rückstandes ziemlich genau angeben, wie viel Schwefelsilber in dem Silber ungefähr bei seinem Frstarrungspunkt gelüst war. Hier waren es $18.46 \%$.

Bei einem Schwefelsilbergehalt von nur $2 \%$, wie bei König I, wird schon sehr viel Silber in Krystallen erstarrt sein, bevor bei langsamem Fallen der Temperatur die zwischen denselben bleibende Lösung gesättigt ist. Eine solche wird schliefslich nur die Fugen zwischen den Silberkrystallen ausfüllen, und bei dem rölligen Erstarren werden sich auch die Schwefelsilberteilchen in diesen Fugen ausscheiden, wie die Schnittfläche des Königs I es deutlich zeigt.

Läge nun der Schmelzpunkt des Schwefelsilber's höher wie der des Silbers, so mülsten sich bei dieser langsamẹn Abscheidung Krystalle von Schwefelsilber bilden können, indem in der noch flüssigen Grundmasse sich jedes ausgeschiedene Teilchen regelmälsig an das vorhergehende anlagert. Dieser Vorgang scheint bei der Abscheidung des im Schwefelsilber gelösten Silbers eingetreten zu sein. Wenigstens deutet die regelmälsige Anordnung einzelner dieser Silberteilchen darauf hin. Leider lassen dieselben sich nicht isolieren.

Das Schwefelsilber aber ist bei seinem tieferliegenden Schmelzpunkt, wälırend es ausgeschieden wird, noch flüssig. So lange nun die Hiussigen Schwefelsilberteilchen von der ebenfalls noch flïssigen Mutterlauge, dem Silber, umgeben sind, werden sie naturgemäl's Tropfenform annehmen und diese auch beim krstarren der sie umgebenden Substanz beibehalten. Allerdings können sie dann durch Einwirkung der bereits erstarrten Silberkrystalle Abdrücke derselben annehmen, wie sie ja auch getunden wurden. Krystalle aber wird das Schwefelsilber in diesem Falle nicht bilden können.

Das Selensilber verhält sich dem Schwefelsilber ganz analıg. Eine Schmelzung von Silber mit 50\%, Selensilber ergah nach dem langs:amen Erkalten einen König, wèlcher ebenfalls eine obere dunkle Schicht und die gröl'seren Einschlüsse zeigte, und mit der Lupe 
auch die kleinen schwarzen Punkte auf der Schnittlïche erkennen liefs. Ein homogen erscheinendes Stück desselben Teiles wurde in verdünnter kalter Salpetersäure (1.1 spez. Gew.) gelöst. $3.6890 \mathrm{~g}$ hinterliefsen $0.8755 \mathrm{~g}=23.7 \%$ Rückstand von demselben Aussehen, wie das ausgelöste Schwefelsilber. Der Rückstand erwies sich als Selensilber. $1.1272 \mathrm{~g}$ ergaben $1.0950 \mathrm{~g} \mathrm{AgCl}$.

$$
\begin{aligned}
\text { Gefunden: } & \text { Berechnet: } \\
73.12^{\circ} \% \mathrm{Ag} & 73.22^{\circ} \% \mathrm{Ag} \text {. }
\end{aligned}
$$

Es wurde hier versucht, den Selengehalt des Selensilbers direkt zu bestimmen. Zu dem Ende wurde das Selensilber in Salpetersäure gelöst und zuerst das Silber ausgefällt. Da, bei dem Verdünnen der Lösung sich selenigsaures Silber ausscheiden würde, so wurde zu der noch konz. Lösung Salzsäure zugegeben, dann erst verdünnt und längere Zeit aufgekocht und so ein selenfreier Chlorsilberniederschlag erhalten. Das Filtrat wurde zur Entfernung der Salpetersäure zweimal mit Kochsalz und etwas Salzsäure zur Trockne gedampft, dann mit Wasser aufgenommen, etwas Salzsäure zugesetat, das Selen in der Hitze durch schweflige Säure gefällt und längere Zeit aufgekocht. Das Filtrat wurde nochmals zur Trockne gedampft und eine kieine Menge Selen aus ihm noch gefällt. Das Selen wurde schliefslich auf einem gewogenen Filter bei $105^{\circ}$ getrocknet und gewogen. Obwobl genau nach Vorschrift gearbeitet wurde, fand sich stets zu wenig Selen. Vier Bestimmungen ergaben:

$22.55 \% \mathrm{Se}$
$24.29 ", "$
$23.45, "$,
$24.75, "$,
im Mittel $23.82 \%$ statt $26.78 \%$.

Um die Richtigkeit der Methode zu prüfen, wurden $0.5164 \mathrm{~g}$ Selen mit Königswasser oxydiert und genau nach Vorschrift behandelt. Es wurden ausgewógen $0.5005 \mathrm{~g} \mathrm{Se}=90.77 \%$ der Einwage. Mit diesen Erfahrungen stimmt überein, was RATHKE in seiner Abhandlung über Selen ${ }^{1}$ über die Bestimmung desselben und die Ungenauigkrit dieser Methode sagt.

Bei den bis jetzt beschriebenen Versuchen ist also die ursprüngliche Absicht, Krystalle von Schwefelsilber und Selensilber im geschmolzenen Silber zu erhalten, nicht erreicht worden. Sie haben

1 Zeitschr. anal. (Yhem. (1870), 9, 484. 
aber gezeigt, dals sich in geschmolzenen Metall Verbindungen von der chemischen Zusammensetzung natürlicher Mineralien, denselben auch in ihren physikalischen Eigenschaften gleichend, abscheiden können. Sie haben gezeigt, warum in dem gegebenen Fall das Schwefelsilber keine Krystalle bilden konnte, und haben überhaupt die Vorgänge bei der Lösung dieser Verbindungen und ihrer $\mathrm{Ab}$ scheidung beim Erstarren des Metalls klargelegt. Sie bildeten so die Grundlage für die folgenden Versuche, bei welchen es gelang, eine Reihe von Verbindungen dadurch in hübschen Krystallen zu erhalten, dals man sie in einer leichter schmelzbaren Muttermasse auskrystallisieren liels. Diese Verbindungen entsprechen teils direkt den natürlichen Vorkommen, wie das Schwefelblei, teils füllen sie Lücken in der Reibe der natürlichen Mineralien aus, wie das in die Gruppe des Pyrits gehörige Arsenplatin und Antimonplatin.

\section{Schwefelblei und Selenblei im Blei.}

Wie oben ausgeführt, war zu erwarten, dals das schwerer als Blei schmelzbare Schwefelblei, falls es von Blei gelöst wurde, in demselben beim langsamen Erkalten auskrystallisiereu würde.

Es wurde Blei in Form ron Bleikörnern mit so riel Schwefel, als einem Gehalt von 10\% Schwefelblei entsprach, gemengt und unter Borax zusammengeschmolzen. Nach dem langsamen Erkalten erfolgte ein König, welcher im Innern eine Druse mit hübschen Würfelchen enthielt. Beim Auflösen in verdünnter kalter Salpetersäure (1.1 spez. Gew.) zeigten sich im oberen Teile des Königs hübsche kleine, treppenförmig aneinandergereihte Würfel.

Ein Bleikönig mit einem $10 \%$ Selenblei entsprechenden Selengehalt hinterliefs ebenfalls beim Behandeln mit verdünnter kalter Salpetersäure hübsche Würfel im oberen Teile des Königs.

Als jedoch versucht wurde, eine gröfsere Menge der Kryställchen für die Analyse auszulösen, trat der Übelstand ein, dal's dieselben bei längerer Einwirkung auch der verdünnten Säure sich zu zersetzen begannen. Besonders rasch wurden die selenhaltigen Kryställchen angegriffen, wie sich denn in noch mehreren Fällen zeigte, dal's die Selenverbindungen der untersuchten Metalle gegen Süuren riel weniger widerstandsfähig sind, als die entsprechenden Schwefelrerbindungen.

Es wurde nun hier mit gutem Erfolg die Auslösung der Kryställchen aus der Muttermasse mittels Elektrolyse angewendet. In 
besonderen Hachen Tiegeln (Fig. 7) wurden $50 \mathrm{~g}$ Blei mit $1 \mathrm{~g}$ Schwefel, resp. $1 \mathrm{~g}$ Selen, gut gemischt, unter Borax zusammengeschmolzen und langsam erkalten lassen. Er erfölgten flache, scheibenförmige Könige, die sich ihrer gröfseren Oberfläche wegen als Anoden besser rerwenden lielsen, wie die in gewöhnlichen konischen Tiegeln erhaltenen. An diese Könige wurde ein starker Kupferdraht angelötet und dieser durch eine Klemmschraube an einem über die Wanne gelegten starken Drabt befestigt (Fig. 8). Zur Vergröfserung der Anodenoberff̈̈che wurden gleichzeitig vier Könige auf diese Weise neben einander in eine rechteckige Glaswanne eingehängt. Die einzelnen Anoden hingen in Thonzellen, um ein Herüberwachsen des an

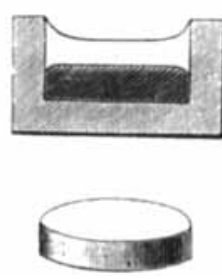

I.ig. 7 .

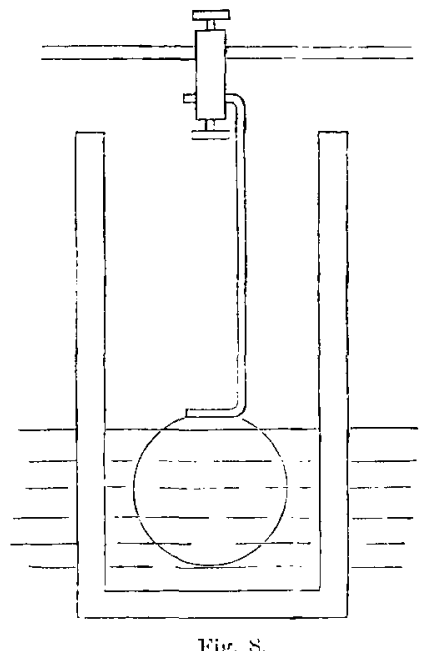

Fis. $:$.

der Kathode ausgeschiedenen Bleies zu verhind ern und um die herabfallenden ausgelösten Kryställchen auffangen zu können. Als Kathode diente ein Stück Bleiblech. Der Abstand der Elektroden betrug etwa $\mathrm{s} \mathrm{cm}$. Der Elektrolyt bestand aus einer Lösung von $50 \mathrm{~g}$ Bleiacetat und $50 \mathrm{~g}$ Natriumacetat in $600 \mathrm{ccm}$ Wasser und $10 \mathrm{ccm}$ freier Essigsäure. Als Stromquelle dienten zwei Akkumulatoren von bis zu 4 Volt Spannung. Die Stromdichte wurde ziemlich niedrig gehalten, etwa $0.2-0.3 \mathrm{Amp}$. auf die Anodenfläche von ca. $10 \mathrm{qcm}$. Es zeigte sich während der Elektrolyse keine Abscheidung von Bleisuperoxyd an der Anode und lösten sich die Kryställchen sehr glatt aus, olme angegriffen zu werden. Es trat nur ein Übelstand auf, nämlich der, dal's sich zwischen den abgefallenen Kryställchen 
kleine Bleibäumchen ausschieden, die sich nur schwer aus der Masse der Kryställchen auslesen lielsen. Es schienen hier die am Boden liegenden Schwefelbleikörperchen der Anode gegenüber als Kathode zu wirken und jene Abscheidungen von Blei zu veranlassen.

Die Schwefelbleikryställchen waren hübsch ausgebildete Würfelchen, teils einzeln oder in unregelmälsigen Gruppen in der Bleimasse sitzend, teils treppenförmig oder zu Nadeln aneinander gereiht (Fig. 9).

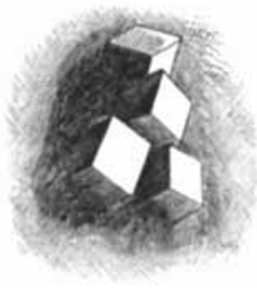

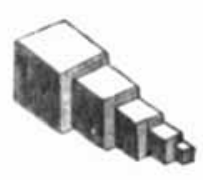

Fig. 9.

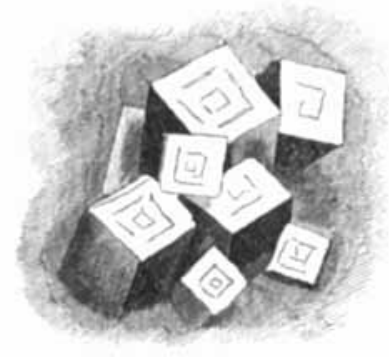

Fig. 10.

Die Analyse ergab die Formel PbS. Sie wurde ausgeführt, indem das feingepulverte Material in einem gewogenen Tiegel mit rauchender Salpetersäure direkt zu Bleisulfat oxydiert und als solches gewogen wurde. $0.2650 \mathrm{~g}$ ergaben $0.3255 \mathrm{~g} \mathrm{PbSO}_{4}$.

$$
\begin{array}{cc}
\text { Gefunden: } & \text { Berechnet: } \\
86.32 \% \mathrm{~Pb} & 86.58 \% \mathrm{~Pb} \text {. }
\end{array}
$$

Die Selenbleikrystalle waren besonders hübsch ausgebildet. Sie zeigten ebenfalls aneinandergereihte Würfel, sowie Gruppen von Würfeln mit vertieften Zeichnungen auf den Flächen, wie die künstlichen Bleiglanzkrystalle zeigen, welche sich manchmal beim Hochofenbetrieb als krystallisierter Ofenbruch bilden. Einige hatten über $1 \mathrm{~mm}$ Durchmesser. Die Analyse ergab die Formel PbSe. In $0.4105 \mathrm{~g}$ fanden sich $0.4375 \mathrm{~g} \mathrm{PbSO}_{4}$.

$$
\begin{array}{cc}
\text { Gefunden: } & \text { Berechnet: } \\
72.770 ! \mathrm{Pb} & 72.28 \% \mathrm{~Pb}
\end{array}
$$

Schon bei diesen Versuchen zeigte es sich, wie schwierig es war, reines Mraterial in genügender Menge für die Analyse zu erhalten, ein Übelstand, der bei den folgenden Versuchen noch zunahm. Denn nur in wenigen Fällen waren die Krystalle grofs genug, um sie einzeln auslesen zu können. Meist handelte es sich 
um ein Gemenge kleiner, immerhin deutlich erkennbarer Krystalle mit einer gröfseren oder geringeren Menge von Verunreinigungen. Bei dem Auslösen mit Säure trat sehr leicht der Fall ein, dafs auch bei ganz dünner Säure die Kryställchen sich mit der Zeit zersetzten und freier Schwefel oder freies Selen sich dem Krystallpulver beimengte. Bei der elektrolytischen Auslösung war oft die Abscheidung von Oxydationsprodukten an der Anode kaum zu vermeiden, welche dann mit den Krystallen einen dicken Schlamm bildeten, ein Fall, der stets bei der Elektrolyse des Wismuts eintrat. Auch fielen leicht kleine Metallteilchen von der Anode ab und blieben ungelöst in dem Schlamm. In den meisten Fällen gelang es mechanisch, durch oftmaliges Schlämmen mit Wasser, am besten auf einem grolsen Uhrglase, die Kryställchen rein zu erhalten. Dabei ging allerdings sehr viel Material verloren und blieb nur wenig für die Analyse. Diese Schwierigkeiten haben jedoch diese Analysen mit denjenigen der meisten natürlichen Mineralien gemein, die ja auch oft durch Einschlüsse und Beimengungen fremder Substanzen verunreinigt sind. Sie haben aber vor diesen den Vorzug, dafs nur die zwei, höchstens drei zur Synthese verwendeten Elemente zu bestimmen sind. Es genügt daher in vielen Fällen die Bestimmung des einen Komponenten, um im Verein mit den äufseren Kennzeichen der künstlichen Krystalle dieselben zu identifizieren. Von dieser Erleichterung wurde besonders bei den selenhaltigen Krystallen Gebrauch gemacht, da die Bestimmung von Selen neben Metallen in derselben Menge Material oft kaum auszuführen ist. Will man das Selen aus der metallhaltigen Lösung mit schwefliger Säure fällen, so reifst dasselbe stets etwas Metall aus der Lösung mit nieder, abgesehen von der früher erwähnten Ungenauigkeit der Bestimmung. Andererseits aber sind die aus einer selenhaltigen Lösung auf irgend eine Art erhaltenen Niederschläge der Metalle sehr oft selenhaltig. Daher wurde in den Fällen, wo sich das Selen aus der Differenz berechnen liefs, auf eine andere Bestimmung desselben verzichtet.

Schwefelwismut und Selenwismut im Wismut.

Bei dem Versuch, Wismut mit Schwefel unter Borax zusammen zu schmelzen, wurde eine dunkle Schlacke erhalten. Es scheint sich hier eine schwefelnatriumhaltige Schlacke gebildet zu haben, welche das Schwefelwismut zu lösen im stande ist, eine Erscheinung, wie sie späterhin ganz ähnlich beim Zusammenschmelzen von Platin und Schwefel unter Borax beobachtet wurde. Wismut und Schwefel 
lassen sich aber sehr wohl in einem bedeckten Tiegelchen zusammenschmelzen, ohne Schwefel zu verlieren. Bei dem niedrigen Schmelzpunkt des Wismuts kann man sogar einfach über einem gewöhnlichen Brenner Wismut in einem Tiegelchen oder Thonschälchen einschmelzen und vorher bereitetes Schwefelwismut eintrageı. Um bei solchen Schmelzungen bei Benutzung des Gasöfchens dem Verbrennen von Schwefel oder Selen nach Möglichkeit vorzubeugen, kann man sich leicht in demselben eine reduzierende Atmosphäre herstellen, indem man den Brenner unter dem Schornstein des Ofens entfernt und die Luftzuführungsöffnung an dem Heizbrenner verkleinert.

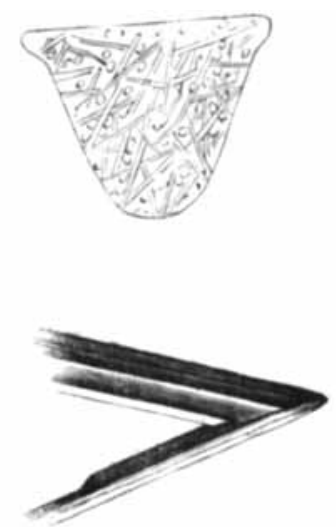

Fig. 11.

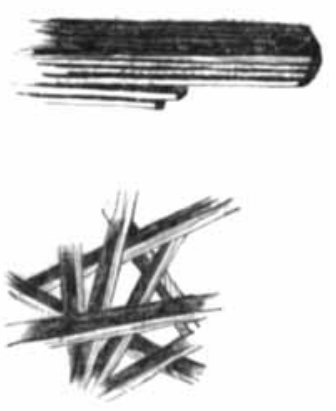

Fig. 12.

Ein Wismutkönig mit $10 \%$ Schwefelwismut war nach dem Erkalten vollständig mit kleinen Nädelchen durchsetzt, welche sich durch Auslösen mit verdünnter kalter Salpetersäure gut isolieren liefsen. Schwefelwismut und Wismut liefsen sich in jedem Verhältnis zusammenschmelzen, ohne dals sich eine besondere Schicht von Schwefelwismut aluschied. Vielmehr durchsetzten bei etwa $50 \%$ Schwefelwismut lange Nadeln den ganzen König und bildeten nach dem Behandeln mit verdünnter kalter Salpetersäure (1.1 spez. Gew.) ein zusammenhängendes Skelett (Fig. 11). Die einzelnen Nadeln hatten das Aussehen des natürlichen Wismutglanzes und waren zu büschelförmigen Aggregaten zusammengewachsen. Es waren auf den Seitenflächen geriefte Säulen, welche aber weder deutliche Endflächen noch glatte Prismenflächen erkennen liefsen (Fig. 12).

Die Analyse ergab in $0.6080 \mathrm{~g} 0.5518 \mathrm{~g} \mathrm{Bi}_{2} \mathrm{O}_{3}$, entsprechend der Formel $\mathrm{Bi}_{2} \mathrm{~S}_{3}$. 
Gefunden:

Berechnet:

$81.36 \% \mathrm{Bi}$

$81.28 \%$ Bi.

Um Selenwismutkryställchen zu erhalten, wurden $10 \mathrm{~g}$ Wismut mit $0.5 \mathrm{~g}$ Selen im bedeckten Tiegel zusammengeschmolzen. Der erhaltene König war aufsen mit einer Kruste Selenmetall überzogen, welche beim Auflösen in kalter verdünnter Salpetersäure (1.1 spez. Gew.) zurückblieb. An der Innenseite dieser Kruste safsen unregelmäfsig durcheinander gewachsene Krystallblättchen von dem Aussehen sechsseitiger rhomboëdrischer Tafeln (Fig. 13). Im Innern des Königs salsen neben solchen Blättchen mehr oder weniger verzerrte reguläre Oktaëder (Fig. 14). Dies führte dazu, auch jene Blättchen als verschobene Oktaëder aufzufassen, wie nachstehende Zeichnung verdenthichen soll (Fig. 15). Jedenfalls stimmt die Krystallform nicht mit der des natürlichen Selenwismuts, welches dem Schwefelwismut isomorph ist, üherein.

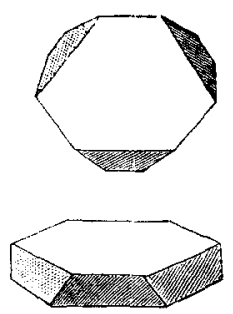

Fi.z. 13.

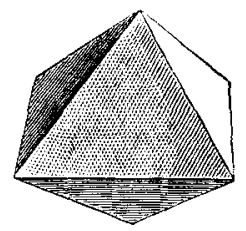

Fig. 14.

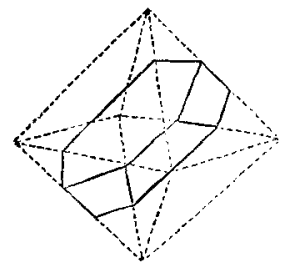

Fig. 15.

Da die Kryställchen sich in verdünnter kalter Salpetersäure rasch unter Abscheidung von rotem Selen zersetzten, so wurde auch hier die elektrolytische Auslösung versucht. Dieselbe war aber bei Wismut mit Schwierigkeiten verknüpft, da die Ausscheidung basischer Salze und die Abscheidung dicker weilser Krusten an der Anode in den meisten Fällen die Auslösung der Kryställchen störte. Eine Lösung von salpetersaurem Wismut in Essigsäure mit der äquivalenten Menge essigsaurem Natron versetzt, erwies sich als der zweckmäfsigste Elektrolyt. Sie blieb beim Verdünnen klar. Jedoch liefs sich im Laufe der Zeit auch durch Zusatz von viel Essigsäure die Abscheidung weifser Krusten an der Anode nicht vermeiden. Die grölseren Teilchen derselben liefsen sich durch Auslesen und Schlämmen, die schlammigen Teile durch Waschen auf einem Sieb cntfernen. Salzsäure löste die weifsen Abscheidungen langsam weg, 
schien aber auch die Kryställchen anzugreifen. Die übrige Anordnung der Elektrolyse war wie bei dem Blei.

Die Analyse der Kryställchen war ebenfalls mit Schwierigkeiten verknüpft. Fällt man das Wismut durch Verdünnen als $\mathrm{BiOCl}$, so erhält man stets einen selenhaltigen Niederschlag. Ebenso, wenn man das Selen durch Schwefelammonium von dem Wismut zu trennen sucht. Fällt man mit Ammoniumkarbonat, so kann man nur durch längeres Kochen mit einem Überschufs des Fällungsmittels einen selenfreien Niederschlag bekommen. Durch den Überschufs des Fällungsmittels wird aber die Genauigkeit der Wismutbestimmung beeinträchtigt. Es wurde auch versucht, durch längeres Kochen und Abdampfen der Wismut und selenige Säure haltenden Lösung mit Schwefelsäure, die selenige Säure, die leichter siedet wie Schwefelsäure, auszutreiben. Dampft man die Lösung in einem Platintiegel ein, so kann man durch starkes Glühen das schwefelsaure Wismut in Oxyd überführen. Ein Versuch mit Material von bekanntem Wismutgehalt ergab auf diese Weise ein um ein Geringes zu niedriges Resultat.

Eine Analyse von möglichst reinem Material ergab in $0.4563 \mathrm{~g}$ $0.4315 \mathrm{~g} \mathrm{Bi}_{2} \mathrm{O}_{3}=84.77 \% \mathrm{Bi}$. Dieser Wismutgehalt würde der Formel $\mathrm{Bi}_{2} \mathrm{Se}$ entsprechen, welche $84.21 \% \mathrm{Bi}$ verlangt. Eine Probe von elektrolytisch ausgelöstem Material, aus welchem sich die letzten geringen Spuren der weifsen Abscheidungen nicht mehr mechanisch entfernen lielsen, ergab in $0.3079 \mathrm{~g}$ Material $0.2963 \mathrm{~g} \mathrm{Bi}_{2} \mathrm{O}_{3}=86.21 \% \mathrm{Bi}$. Eine zweite Probe desselben Materials, welche zur Entfernung jener Verunreinigungen mit verdünnter Salzsäure behandelt war, ergab in $0.4567 \mathrm{~g} 0.4147 \mathrm{~g} \mathrm{Bi}_{2} \mathrm{O}_{3}=81.41 \% \mathrm{Bi}$.

Beide Resultate liegen in der Nähe von $84.21 \%$ und weit ab von $63 \%$, welchen Prozentsatz die Formel $\mathrm{Bi}_{2} \mathrm{Se}_{3}$ verlangen würde. Jedoch kann man die Formel $\mathrm{Bi}_{2} \mathrm{Se}$ nicht für bestimmt erwiesen annehmen. Jedenfalls entspricht die Verbindung weder in ihrer Krystallform noch in ihrer chemischen Zusammensetzung dem naturlichen Selenwismut mit der Formel $\mathrm{Bi}_{2} \mathrm{Se}_{3}$.

Schwefelsilberwismut und Selensilber in Wismut.

Da Wismut sich als sehr gutes Krystallisationsmittel erwiesen hatte, wurde versucht, auch Schwefelsilber und Selensilber, welche ja im Silber nicht krystallisieren, in dem leicht schmelzbaren Metall zum Krystallisieren zu bringen, nachdem Versuche, sie in Zink und Aluminium zu lösen, fehlgeschlagen waren. 
Es wurden in einem bedeckten Tiegel $20 \mathrm{~g}$ Wismut ohne Borax eingeschmolzen und $2 \mathrm{~g}$ Schwefelsilber in dasselbe eingetragen. Beim Auflösen des langsam erkalteten Königs in kalter verdünuter Silpetersäure (1.1 spez. Gew.) zeigten sich zuerst dunkle Kryställchen, untermischt mit silberweifsen, und liels sich in der Lüsung kein Silber nachweisen. Mit der Zeit lösten sich die hellen Kryställchen, welche wohl Silber oder eine Wismutsilberlegierung waren, auf, und hinterblieben nur die dunklen Kryställchen, die beim Trocknen einen stahlblauen Glanz annahmen. Die Elektrolyse hinterliefs ein Gemisch von dunklen und hellen Kryställchen. Beide bildeten hübsche Gruppen von aneinander gereihten Oktaëdern (Fig. 16). Die dunklen Kryställchen enthielten Silber, Wismut und Schwefel. Die Analyse wurde ausgeführt, indem das Material in verdünnter Salpetersäure

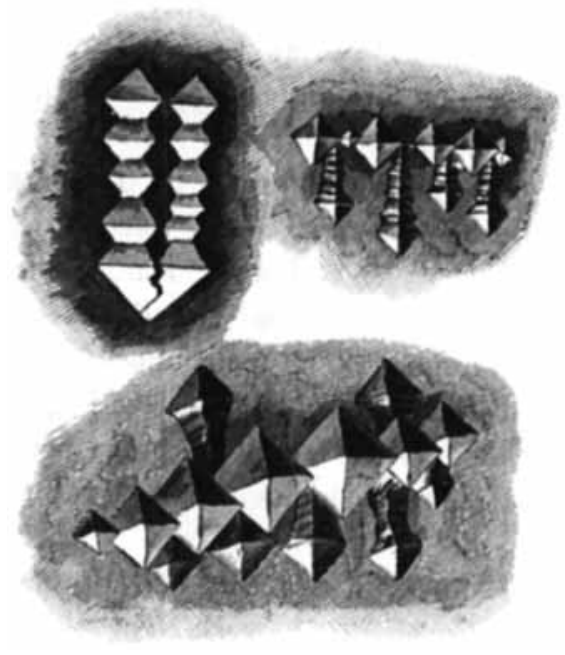

Fis. 16. gelöst wurde. Zunächst wurde, ohne stärker zu verdünnen, das Silber als Chlorsilber gefällt. Der Niederschlag wurde nach dem Abfiltrieren der meisten Flüssigkeit mit verdünnter Salpetersäure ausgekocht und das Filter mit heilser verdünnter Salpetersäure ausgewaschen, um zu rermeiden, dals $\mathrm{BiOCl}$ beim Niederschlag blieb. Dann wurde das Wismut durch Verdünnen gefällt, der Niederschlag wieder in Salpetersäure gelöst, mit Schwefelwasserstoff gefällt und als $\mathrm{Bi}_{2} \mathrm{~S}_{3}$ auf gewogenem und bei $100^{\circ}$ getrocknetem Filter gewogen. Zuletzt wurde der Schwefel als schwefelsaures Baryum bestimmt. Die Analyse ergab ziemlich annähernd die Formel $\mathrm{AgBiS}_{2}$ oder $\mathrm{Ag}_{2} \mathrm{~S}+\mathrm{Bi}_{2} \mathrm{~S}_{3}$, nümlich in $0.6335 \mathrm{~g}$ Substanz $0.4100 \mathrm{~g}$ $\mathrm{Bi}_{2} \mathrm{~S}_{3}$ und $0.2560 \mathrm{~g} \mathrm{AgCl}$, in $0.6300 \mathrm{~g} 0.7383 \mathrm{~g} \mathrm{BaSO}_{4}$.

Gefunden:
$52.77^{\circ}, \mathrm{Bi}$
$30.32, \mathrm{Ag}$
$16.11, \mathrm{~s}$
$99.20^{\circ}:$

Gefunden: $32.77^{\circ} \% \mathrm{Bi}$ $30.32, \mathrm{Ag}$ $99.20^{\circ}$ 。
Berechnet: $54.74 \% \mathrm{Bi}$ $28.42, \mathrm{Ag}$ $16.84, \mathrm{~S}$ 
Die Kryställchen entsprechen also dem natürlichen Silberwismutglanz in ihrer chemischen Zusammensetzung, jedoch nicht in ibrer Krystallform, da derselbe rhombisch krystallisiert.

Nun wurden ebenso $2 \mathrm{~g}$ Selensilber in $20 \mathrm{~g}$ Wismut in einem bedeckten Tiegel eingeschmolzen. Beim Auflösen des langsam erkalteten Königs in verdünnter kalter Salpetersäure, ebenso wie bei der Elektrolyse in essigsaurer Lösung, hinterblieben dunkle Kryställchen von eigentümlicher federartiger Form, im Gegensatz zu dem vorhergehenden Versuch ohne Beimengung heller Kryställchen. Unter dem Mikroskop stellten sie sich als federartig aneinander gereihte vierseitige Säulen mit pyramidalen Endẗ̈̈chen heraus. Dazwischen zeigten sich aber auch kleine, rundum ausgebildete Kryställchen, welche Rhombendodekaëder zu sein schienen. Denselben Eindruck machten die Säulchen von oben betrachtet. Man kann daher wohl auch diese als in der Richtung einer Hauptaxe in die Länge gezogene Rhombendodekaëder auffassen. Da, wie die Analyse zeigte, hier Kryställchen von Selensilber vorlagen, stimmt diese Annahme damit überein, dafs MargotTet auch auf anderem Wege Selensilber in Rhomben. dodekaëdern erhielt. ${ }^{1}$ Auch findet sich bei dem natürlichen Schwefelsilber die hier beobachtete, säulenförmige Ausbildung und federartige Anordnung.

Die Kryställchen erwiesen sich völlig frei von Wismut und ergaben den der Formel $\mathrm{Ag}_{2} \mathrm{Se}$ entsprechenden Silbergehalt, nämlich in $0.5675 \mathrm{~g} 0.5495 \mathrm{~g} \mathrm{AgCl}$.

Berechnet: $73.22 \%$ Ag.
Gefunden: $72.88^{\circ} \% \mathrm{Ag}$.
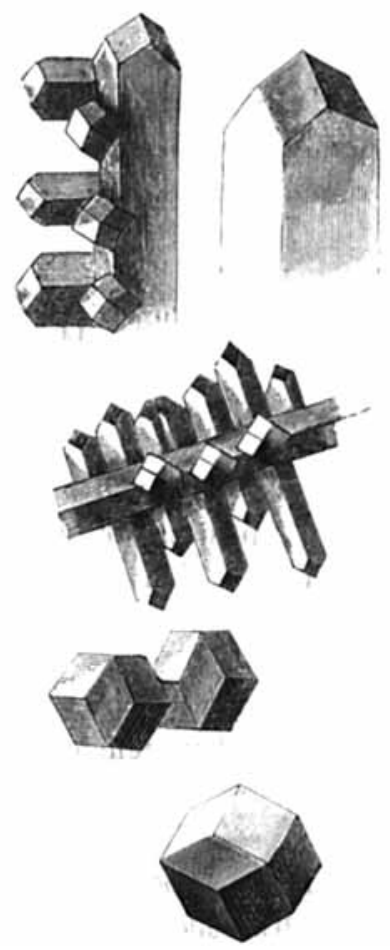

Fig. 17.

Sehr auffallend ist das verschiedene Verhalten des Schwefelsilbers und Selensilbers beim Einschmelzen in Wismut. Denn während bei dem ersteren sich offenbar ein Teil des Silbers gegen Wismut austauscht, so dals eine Doppelverbindung entsteht, wird

1 Compl. rend. SJ, 1142.

Z. anorg. Chem. IX. 
das Selensilber unverändert von dem Wismut gelöst und als solches wieder ausgeschieden.

Um auch Kryställchen von der Formel $\mathrm{AgBiSe}_{2}$ analog dem Silberwismutglanz zu erhalten, wurde zunächst Silber und Wismut mit einem geringen Selenüberschufs entsprechend jener Formel zusammengeschmolzen. Der erhaltene König hatte das berechnete Gewicht. Von demselben wurden $3 \mathrm{~g}$ in $30 \mathrm{~g}$ Wismut aufgelöst. Beim Auflösen des langsam erkalteten Wismutkönigs mittels Elektrolyse hinterblieb ein einheitlich aussehendes Krystallpulver. Unter dem Mikroskop zeigten sich hübsche glänzende, dunkelgraue Oktaëderchen, an die Schwefelsilberwismutkrystalle erinnernd. Die Analyse ergab jedoch nicht die diesen entsprechende Zusammensetzung, vielmehr stimmte dieselbe annähernd mit der Formel einer Mischung von $\mathrm{xBi}_{2} \mathrm{Se}+\mathrm{yAg}_{2} \mathrm{Se}$ überein. Das Verhältnis von $\mathrm{x}: \mathrm{y}$ ist ziemlich genau $6: 1$, wofür auch die nebenstehenden Zahlen berechnet sind. Es fanden sich in $0.8110 \mathrm{~g}$ Material $0.6960 \mathrm{~g} \mathrm{Bi}_{2} \mathrm{O}_{3}$ und $0.0770 \mathrm{~g} \mathrm{AgCl}$.

\begin{tabular}{cr} 
Gefunden: & Berechnet: \\
$76.91 \% \mathrm{Bi}$ & $76.50 \% \mathrm{Bi}$ \\
$7.14 \% \mathrm{Ag}$ & $6.60 \% \mathrm{Ag}$ \\
$15.95 \%$ Se (Differenz) & $16.90 \% \mathrm{Se}$ \\
\hline $100.00 \%$ & $100.00 \%$
\end{tabular}

Dafs sich auch hier Wismut und Selen in dem Verhältnis $2: 1$ finden, spricht für die Wahrscheinlichkeit der für die oben beschriebene Selenwismutrerbindung angenommenen Formel $\mathrm{Bi}_{2} \mathrm{Se}$.

Kupferverbindungen.

Auch mit Schwefelkupfer und Selenkupfer wurden eine Reihe von Versuchen gemacht, die aber nicht so eingehend verfolgt wurden.

Der im Kupfer enthaltene Schwefel scheidet sich in demselben als Halbschwefelkupfer aus, wie das Schwefelsilber im Silber. So enthält der Anodenschlamm von der elektrolytischen Kupferraffiuation Halbschwefelkupfer.

Halbschwefelkupfer wurde in Blei aufgelöst und schied sich in demselben beim Iangsamen Erkalten in Gestalt von hübschen Oktaëderchen aus, die aber auch etwas Blei enthielten. Es wurde hier die Erfahrung bestätigt, dafs, während das natürliche Halbschwefe]kupfer, der Kupferglanz, rhombisch krystallisiert, die künstlichen Krystalle desselben regulär sind. ${ }^{1}$

1 Tschermak, Mineralogie, S. 353. 
Beim Auflösen von Halbselenkupfer in Blei und Behandeln des Bleikönigs mit verdünnter kalter Salpetersäure hinterblieb ein buntes Gemenge verschiedenartiger Krystalle. Unter dem Mikroskop zeigten sich neben rotgelben und stahlblau angelaufenen Oktaëderchen und dunklen federförmigen Kryställchen sonderbare kupferfarbene Gebilde von der Form aneinander gereihter rhombischer Blättchen (Fig. 18).

Es wurde auch versucht, Doppelverbindungen des Kupfers und Wismuts, sowie des Bleies und Wismuts mit Schwefel oder Selen herzustellen. Hierzu wurden zuerst Gemenge der drei Elemente nach der Zusammensetzung natürlich vorkommender Mineralien, entsprechend z. B. dem Zorgit $\mathrm{PbSe}+\mathrm{Cu}_{2} \mathrm{Se}$ oder dem Emplektit $\mathrm{Cu}_{2} \mathrm{~S}+\mathrm{Bi}_{2} \mathrm{~S}_{3}$, geschmolzen, und diese dann in Blei oder Wismut eingetragen. Die beim Auflösen der Könige zurückbleibenden Kryställchen waren meist nicht einheitlich. So entstand statt der Verbindung $\mathrm{CuBiS}_{2}$ ein Gemenge von Nädelchen und kleinen Oktaëdern, statt der Verbindung $\mathrm{PbBi}_{2} \mathrm{~S}_{4}$ oder $\mathrm{PbBi}_{2} \mathrm{Se}_{4}$ ein Gemenge von Würfelchen, offenbar Schwefel- oder Selenblei, und silberglänzenden Blättchen.

Nur eine Selenkupferwismutverbindung lieferte einheitliche Oktaëderchen, die wahrscheinlich den beschriebenen Selensilberwismutkryställchen entsprechen, deren Analyse aber keine Formel berechnen liefs.

Ein Versuch, eine dem Rotgültigerz entsprechende Verbindung im Antimon krystallisieren zulassen, gelang nicht. Auch Schwefelantimon krystallisierte nicht im Antimon aus, vielmehr setzte sich das ganze Schwefelmetall als gesonderte Schicht über dem unverändert bleibenden Antimon ab.

Thallium mit einem Überschufs von Schwefel geschmolzen gab eine schwarze, strahlig krystallinische Masse. Ein Teil davon wurde in eine grölsere Menge Thallium eingeschmolzen. Der erhaltene König löste sich jedoch in verdünnter kalter Salpetersäure (1.1 spez. Gew.) auf, ohne Kryställchen zu hinterlassen.

Wenn auch von diesen letzten Versuchen nur wenige ein direktes Resultat gegeben haben, was wohl zum gröfsten Teil daran liegt, dafs es an Zeit mangelte, sie alle eingehend $z u$ verfolgen, so zeigen doch mehrere derselben die Möglichkeit, auch komplizierter zusammengesetzte Sulfide und Selenide in den Metallen krystallisieren zu lassen. Man wird hier sicher mit dieser Methode ein 
gutes Resultat erzielen können, wenn man durch eine gröfsere Zahl von Versuchen das richtige Verhältnis, in welchem die Elemente zur Darstellung der betreffenden Verbindung zusammengeschmolzen werden müssen, sowie das für jeden Fall am besten als Lösungsmittel zu verwendende Metall ausprobiert hat. Dann kann man jedenfalls auch durch Variation der Auslösungsmethoden, durch Anwendung verschiedener Säuren von wechselnder Stärke, durch Elektrolyse in verschiedenen Laugen, aus einem Gemenge von mehreren Arten von Kryställchen eine Sorte derselben isolieren.

Bevor zu dem nächsten Teil dieser Untersuchungen übergegangen wird, welcher eine Anzahl Verbindungen des Platins und Palladiums behandeln soll, mögen hier noch einige nicht uninteressante Beobachtungen, welche im Laufe der Versuche mit Silber, Schwefelsilber und Selensilber gemacht wurden, eine beiläufige Erwähnung finden.

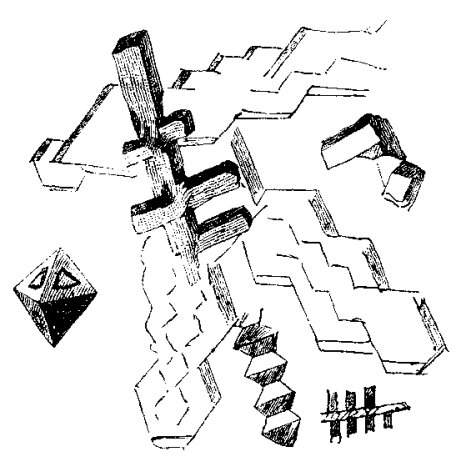

Fig. 18.

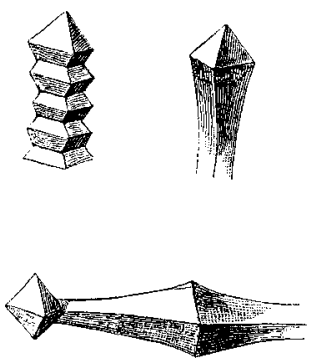

Fig. 19 .

Beim Nischen von Silberkryställchen mit pulverförmigem Selen batte ich mehrfach beobachtet, dals die Silberkryställchen sich bald schwarz färbten, so dals nach längerem Mischen überhaupt keine helleren Kryställchen in dem Gemisch mehr zu erkennen waren. Es wurden nun Silberpulver und Selen (wenig mehr als der Formel $\mathrm{Ag}_{2} \mathrm{Se}$ entsprach) in einer Reibschale zusammen gerieben und dabei nach kurzer Zeit ein einheitliches schwarzes Pulver erhalten. Dasselbe wurde, um überschüssiges Selen zu entfernen, mehrmals längere Zeit mit Schwefelkohlenstoff behandelt, mit Alkohol gewaschen und getrocknet. Verdünnte Salpetersäure wirkte nicht auf dasselbe ein. Eine Analyse ergab $73.86 \%$ Ag. Die Formel $\mathrm{Ag}_{2} \mathrm{Se}$ verlangt $73.22 \% \mathrm{Ag}$. Dafs hier wirklich eine Einwirkung von Selen auf Silber bei gewöhnlicher Temperatur unter Bildung von $\mathrm{Ag}_{2} \mathrm{Se}$ 
stattgefunden hat, scheint demnach wahrscheinlich, kann aber nicht als bestimmt erwiesen hingestellt werden.

Um das Schwefelsilber aus dem Silber herauszulösen, wurde, wie dort erwähnt, auch die Elektrolyse zu Hilfe genommen. Dabei wurde in einer Lauge von salpetersaurem Silber die Bildung von besonders hübschen Silbersuperoxydkrystallen, welches an sich ja bekannt ist, beobachtet. Es zeigte sich, dafs dieselbe besonders bei stark saurer Lauge und sehr hoher Stromdichte eintrat. Da bei den betreffenden Versuchen die Anoden sehr klein waren, so betrug die Stromdichte oft mehrere Tausend Ampères auf den Quadratmeter der Anodenoberfläche. Dabei wuchsen lange Nadeln von Silbersuperoxyd an den Kanten der Anode und an dem diese tragenden Platindraht aus. Dieselben bestanden aus aneinandergereihten Oktaëdern, die aber meist nicht einzeln $\mathrm{zu}$ erkennen waren, so dafs die Krystalle sonderbar in die Länge gezogen schienen (Fig. 19).

\section{Schwefel- und Selenverbindungen des Platins und Palladiums.}

Eine dem Auftreten von Selensilber im geschmolzenen Silber analoge Erscheinung wurde bei der Verarbeitung der aus der Goldscheidung stammenden platinhaltigen Rückstände beobachtet. Bei der Auflösung des güldigen Silbers in Schwefelsäure hinterbleibt ein meist aus Gold bestehender Schlamm, welcher auch alle im Scheidegut enthaltenen Platinmetalle enthält. Aufserdem sammeln sich aber in demselben eine ganze Reihe anderer Elemente, wie Selen, Arsen, Antimon, Wismut und hier und da wohl auch seltenere Elemente, welche durch Verarbeitung unreinen Materials, wie z. B. der Anodenschlämme aus der Kupferelektrolyse von Mansfeld, in den Betrieb kommen, an. Daher weist die weitere Behandlung dieses Schlammes, behufs Reindarstellung von Gold, Platin und Palladium, eine Fülle von interessanten Erscheinungen auf, deren mehrere im Lauf der folgenden Untersuchungen Erwähnung finden werden.

So bleibt beim Auflösen selenhaltigen Silbers das Selen als Selensilber im Goldschlamm. Beim Lösen desselben in Königswasser wird es mit aufgelöst, und nur ein geringer Teil des Selens fällt mit dem Gold bei dessen Fällung durch Eisenchlorür aus. Die entstandene Eisenchloridlauge wird durch Eisen in Chlorür zurückverwandelt und fallen dabei die Platinmetalle, zugleich aber auch Selen und alle verunreinigenden Metalle, als schwarzer Schlamm aus. 
Wird derselbe unter Fluls zusammengeschmolzen, so schmilzt er viel leichter, als der hohe Gehalt an Platinmetallen erwarten lärst. Beim Auflösen des erhaltenen Metallkönigs hinterbleiben dann oft im Königswasser unlösliche metallglänzende Flitterchen, die sich bei näherer Untersuchung als Selenplatin erweisen.

Es lagen mir solche in früheren Jahren zufällig erhaltene Körperchen zur Untersuchung vor. Unter dem Mikroskop liefsen sich einzelne säulenförmige Kryställchen erkennen. Jedoch zeigten die meisten Körperchen keine bestimmbare Krystallform, sondern machten den Eindruck von Bruchstücken und Splittern grölserer Individuen.

Eine Analyse der Körperchen schien schwierig, da sie sich weder in Königswasser, noch in Salzsäure und chlorsaurem Kali lösten. I)a aber eine qualitative Prüfung neben Platin und wenig Palladium nur Selen erkennen lief's, so erschien der einfachste Weg der Analyse, das Selen durch Glühen an der Luft zu verjagen. Nach dem Glühen löste sich der Rückstand völlig in Königswasser. Platin und Palladium wurden hier, wie in allen späteren Fällen, folgendermal'sen neben einander bestimmt. Die Königswasserlösung, zu welcher nur die eben genügende Menge Salpetersäure verwendet worden war, wurde mit Wasser und etwas Salzsäure nochmals abgedampft, um sicher alles Palladium als Chlorïr in der Lösung zu haben. In der möglichst eingeengten Lösung wurde das Platin durch Zusatz von festem Salmiak gefällt. Der nach 24 Stunden abfiltrierte Niederschlag wurde zuerst mit konz. Salmiaklösung und dann mit 90\% igem Alkohol ausgewaschen. Im Filtrat kann man das Palladium als Palladosamminchlorid fällen. Besser aber ist es, dasselbe aus der möglichst wieder eingeengten Lösung als Palladiumsalmiak abzuscheiden, indem man dieselbe, event. unter nochmaligem Zusatz von etwas Salmiak, mit wenig konz. Salpetersäure aufkocht. Es fällt dann in konz. Salmiaklösung und $90 \%$ igem Alkohol unlöslicher Palladiumsalmiak. Beide Niederschläge werden durch vorsichtiges Glühen in Metall übergeführt und als solches gewogen. Auf diese Weise ergaben $0.4290 \mathrm{~g}$ der Körperchen $0.2982 \mathrm{~g} \mathrm{Pt}$ und $0.0065 \mathrm{~g} \mathrm{Pd}$ entsprechend der Formel PtSe, worin etwas Pt clurch $P d$ vertreten ist.

$\begin{array}{ll}\text { Gefunden: } & \text { Berechnet: } \\ 69.50 \% \mathrm{Pt} & 71.17 \% \mathrm{Ht} \\ 1.52 \% \mathrm{Pd} & \\ 71.02 \% \text { Platinmetall. } & \end{array}$


Der obenerwähnte, hauptsächlich aus Platinmetallen bestehende schwarze Schlamm wird zur Gewinnung derselben wieder in Königswasser gelöst und zuerst Platin durch Salmiak ausgefällt. Aus der Mutterlauge wird das Palladium als Palladosamminchlorid durch Fällen mit Ammoniak, Lösen des Niederschlags im Überschufs und Fällen mit Salzsäure gewonnen. Dasselbe wird durch Glühen in Palladiumschwamm übergeführt. In früheren Jahren wurde dabei einmal beobachtet, dals dieses Palladosamminchlorid beim Glühen keinen gesinterten Schwamm, sondern eine geschmolzene Masse hinterliefs, aus welcher sich durch Königswasser glänzende Flitterchen von der Zusammensetzung PdSe berauslösen liefsen. ${ }^{1}$ Hier hatte offenbar der Gehalt an PdSe den Schmelzpunkt des Palladiums erniedrigt.

Die mir vorliegenden Selenplatinkörperchen waren offenbar krystallisiert. Es muls daher angenommen werden, dals neben Selen noch andere Verunreinigungen in dem damals verarbeiteten Platinschlamm waren, welche mit dem übrigen Teil der Platinmetalle eine leicht schmelzbare, dem Auskrystallisieren der Selenverbindung günstige Muttermasse bildeten, welche durch Königswasser aufgelöst wurde. Da jedoch die Zusammensetzung derselben nicht mehr zu ermitteln war, mufste zunächst aufgegeben werden, Krystalle von Selenplatin oder Selenpalladium synthetisch zu erhalten. Denn wollte man, was ja am nächsten lag, Selenpalladium in Palladium einschmelzen, so war zu erwarten, daís die leichter schmelzbare Verbindung in dem schwerer schmelzbaren Metall keine Krystalle bilden würde, eine Erwartung, welche auch durch den Versuch bestätigt wurde.

Immerhin aber schienen die hier in Frage kommenden Verbindungen auch in amorphem Zustande einer näheren Untersuchung wert, besonders da über Selenverbindungen des Platins und Palladiums fast gar nichts bekannt ist.

\section{Palladiumsubsulfür $\mathrm{Pd}_{2} \mathrm{~S}$.}

Nach SchNeIDER ${ }^{2}$ wird dasselbe beim Zusammenschmelzen von 1 Teil Palladosamminchlorid, 6 Teilen Pottasche, 6 Teilen Schwefel und 3 Teilen Salmiak als grauer spröder Regulus erhalten.

Im vorliegenden Fall wurden $10 \mathrm{~g}$ Palladosamminchlorid mit $2.5 \mathrm{~g}$ Schwefel (genügend zur Bildung von $\mathrm{PdS}$ ) innig gemischt

\footnotetext{
${ }^{1}$ H. Roessrer, Lieb. Ann. 180, 240.

${ }^{2}$ P'ogy. Ann. 141, 530.
} 
unter einer Boraxdecke zusammengeschmolzen. Im Gasofen ohne Gebläse wurde bei etwa Goldschmelzhitze ein Metallkönig erhalten, welcher auf seiner Oberfläche krystallinische Zeichnungen zeigte. Derselbe war graugelb, etwa wie Schwefelnickel, sehr spröde und so hart, dafs er kaum von Stahl geritzt wurde. Der Bruch war körnig und metallglänzend.

Auch sehr feir gepulvert war die Verbindung in Königswasser nicht völlig löslich. Zum Zweck der Analyse wurde daher das feingepulverte Material mit Soda und Salpeter geschmolzen und der ausgelöste Rückstand mit Königswasser behandelt. Ein dabei noch ungelöst bleibender Rückstand war erst nach wiederholtem Glühen völlig in Königswasser löslich. Aus der Lösung wurde das Palladium als Palladosamminchlorid gefällt, vorsichtig geglüht und als Metall ausgewogen. Die Analyse ergab die Formel $\operatorname{Pd}_{2} \mathrm{~S}$, nämlich in $0.4961 \mathrm{~g}$ Material $0.4332 \mathrm{~g} \mathrm{Pd}$.

$\begin{array}{cr}\text { Gefunden: } & \text { Berechnet: } \\ 87.46 \% \mathrm{Pd} & 86.89 \% \mathrm{Pd}\end{array}$

Nun wurde pulverförmiges Palladium, erhalten durch vorsichtiges Glühen des Palladosamminchlorids, mit ca. $10 \%$ Palladiumsubsulfür gut gemischt. Dieselben wurden unter einer Glasdecke, da Borax bei der grofsen Hitze ảie Tiegelchen zerstörte, im Devilueschen Koksofen mit Gebläse zusammengeschmolzen. Die geschmolzene Masse hinterliefs beim Auflösen in kalter konz. Salpetersäure kleine graue, metallglänzende Körperchen, wie zu erwarten war, ohne Krystallformen, unter dem Mikroskop etwa von dem Aussehen der Fig. 20. natürlichen Platinerze (Fig. 20).

Die Körperchen hatten ebenfalls die Zusammensetzung $\mathrm{Pd}_{2} \mathrm{~S}$. Es fanden sich in $0.1300 \mathrm{~g}$ Material $0.1142 \mathrm{~g} \mathrm{Pd}$.

$$
\begin{array}{cc}
\text { Gefunden: } & \text { Berechnet: } \\
87.79 \% \mathrm{Pd} & 86.89 \% \mathrm{Pd} .
\end{array}
$$

Palladiumselenür PdSe.

Nach Berzelius ${ }^{1}$ vereinigen sich Palladium und Selen unter Wärmeentwickelung zu einer grauen, nicht schmelzbaren Verbindung. Dies ist aufser der Angabe ron H. Rorssher über die schon

I Gmelin-KraUt, 2, 1232. 
erwähnten Selenpalladiumkörperchen das einzige, was sich über Selenpalladium auch in den neuesten Handbüchern findet.

$10 \mathrm{~g}$ Palladosamminchlorid wurden mit $5 \mathrm{~g}$ Selen gut gemischt. Dieselben schmolzen unter Borax im Gasofen ohne Gebläse zu einem glatten spröden König zusammen, welcher etwas heller und nicht ganz so hart war wie der Schwefelpalladiumkönig. Auf dem Bruch war er feinkörnig und metallglänzend. Er zeichnete sich im Gegensatz zu der angeführten Angabe durch seine leichte Schmelzbarkeit aus, indem er leichter wie Silber schmolz, ohne dabei an der Luft Selen zu verlieren. Im Gegensatz zu der Schwefelpalladiumverbindung löste sich das Selenpalladium vollkommen in kochendem Königswasser auf, was die früher gemachte Beobachtung ergänzt, dafs Selenverbindungen oft weniger widerstandsfähig gegen Säuren sind, als die entsprechenden Schwefelverbindungen.

Bei der Analyse machte die Trennung von Palladium und Selen einige Schwierigkeiten. Bei der Ausfällung des Palladiums als Palladosamminchlorid fällt etwas Selen mit, wie schon bei der Beschreibung der zufällig entstandenen Selenpalladiumkörperchen erwähnt wurde. Ebenso fällt Selen mit, wenn man Palladium durch Reduktionsmittel als Metall niederschlagen will. Auch die Elektrolyse aus salzsaurer Lösung lieferte einen selenhaltigen Niederschlag, welcher beim Glühen zusammenschmolz. Bei dem Versuch, das Selen durch Glühen an der Luft zu vertreiben, schmolz die Verbindung zusammen, ohne Selen zu verlieren. Ebenso wurde der Versuch, die Trennung durch Schmelzen mit Soda und Salpeter herbeizuführen, durch Schmelzen des Selenpalladiums verhindert. Nur durch Fällen als roter Palladiumsalmiak wurde ein selenfreier Niederschlag erhalten. $\mathrm{Zu}$ diesem Zweck wurde das feingepulverte Naterial in der eben genügenden Menge Königswasser gelöst, mit festem Salmiak im Überschufs versetzt und nochmals mit etwas Salpetersäure zum Kochen erhitzt.

Hier wie auch bei mehreren der folgenden Platin-, Palladiumund Goldverbindungen erwies es sich als sehr zweckmälsig, vor der genauen Analyse eine orientierende Bestimmung auf trockenem Wege zu machen, da dieselbe in sehr kurzer Zeit auszuführen ist. Es wurde zu diesem Zweck eine gewogene Menge des Materials mit einer bestimmten Menge Feinsilber mit Blei auf der Kupelle abgetrieben. Dann wurde, nachdem nach den Regeln für die Ausführung der trockenen Proben die erfahrungsmälsig durch den Kupellenzug verloren gehende Menge Silber wieder zugezählt war, 
Platinmetall + Silber zusammen ausgewogen. Will man eine genaue Bestimmung auf trockenem Wege erzielen, so hat man nur das Silberkorn in konz. Schwefelsäure aufzulösen und das zurückbleibende Platinmetall zu wiegen. In manchen Fällen, z. B. bei Goldverbindungen, ist eine trockene Probe oft vorzuzieben.

Eine trockene Probe ergab 57\% Pd, die Analyse ergab die Formel PdSe, nämiich in $0.2480 \mathrm{~g}$ Material $0.1407 \mathrm{~g} \mathrm{Pd}$.

$$
\begin{array}{ll}
\text { Gefunden: } & \text { Berechnet: } \\
56.73 \% \text { Pd } & 57.29 \% \text { Pd. }
\end{array}
$$

Um auch im geschmolzenen Palladium Körperchen von Selenpalladium zu erhalten, wurden $60 \mathrm{~g}$ Palladosamminchlorid mit $2 \mathrm{~g}$ Selen unter einer Glasdecke im gewöhnlichen Koksofen bei starker Hitze zusammengeschmolzen und im Ofen langsam erkalten lassen. Die im Palladium gelöste Selenverbindung (etwa $12 \%$ PdSe entsprechend) erniedrigte hier bedeutend dessen Schmelzpunkt, denn reines Palladium wäre bei der angewendeten Hitze nicht geschmolzen. Es erfolgte ein gut geschmolzener König mit krystallinischen Zeichnungen auf der Oberfläche, im Innern von kleinen Hohlräumen durchsetzt, welche offenbar von der Gasentwickelung bei der Zersetzung des Palladosamminchlorids herrührten. Beim Auflösen in kilter konz. Salpetersäure hinterblieben graue, spröde, unregelmäfsige Körperchen von demselben Aussehen wie Schwefelpalladiumkörperchen. Ihr Palladiumgehalt stimmte jedoch weder mit der Formel $P d S e$ noch $\mathrm{Pd}_{2}$ Se überein. Vielmehr fanden sich in $0.2648 \mathrm{~g} 0.2243 \mathrm{~g}$ $=84.69 \% \mathrm{Pd}$. Da angenommen wurde, dals noch metallisches Palladium den Körperchen beigemengt sei, wurden dieselben gepulvert und nochmals mit konz. Salpetersäure behandelt. Die Analyse ergab dasselbe Resultat, nämlich in $0.2225 \mathrm{~g} 0.1882 \mathrm{~g} \mathrm{Pd}$ $=84.78 \% \mathrm{Pd}$. Dieser Gehalt würde einer Formel $\mathrm{Pd}_{4}$ Se entsprechen.

$$
\begin{array}{cc}
\text { Gefunden: } & \text { Berechnet: } \\
84.78 \% \text { Pd } & 84.29 \% \text { Pd. }
\end{array}
$$

So abnorm diese Zusammensetzung im ersten Augenblick erscheint, so hat sie doch eine Analogie in dem Verhalten des Nickels und Kobalts bei der Pla't'Tnerschen Lötrohrprobe. Dort verlieren $\mathrm{CoAs}_{2}$ und $\mathrm{NiAs}_{2}$ beim Schmelzen drei Viertel ihres Arsengehaltes, halten aber das letzte Viertel hartnäckig fest, indem sie $\mathrm{Co}_{2} \mathrm{As}$ und $\mathrm{Ni}_{2} \mathrm{As}$ bilden. Ebenso mufs man annehmen, dafs das Selenpalladium PdSe drei Viertel seines Se-Gehaltes an das überschüssige Palladium unter Bildung von $\operatorname{Pd}_{4}$ Se abgegeben hat. 
Es wurden nun $25 \mathrm{~g}$ Platinpulver, mit $1 \mathrm{~g}$ Selen gut gemischt, unter einer Sanddecke im Devilue-Ofen eingeschmolzen. Es erfolgte ein teilweise geschmolzener König, welcher beim Auflösen in kaltem Königswasser hellglänzende Körperchen hinterliefs, welche den aus Palladium erhaltenen Selenpalladiumkörperchen ähnlich sahen. Einige jedoch erschienen gesplittert, manchmal mit glänzenden Spaltflächen, was ja mit der am Selenplatin beobachteten Spaltbarkeit übereinstimmen würde. Eine Formel liefs sich für dieselben nicht feststellen. Die Analyse ergab in $0.1840 \mathrm{~g} 0.1475 \mathrm{~g} \mathrm{Pt}$ $=80.16 \% \mathrm{Pt}$.

Bei dem Versuch, $\mathrm{Pd}_{2} \mathrm{~S}$ und $\mathrm{PdSe}$ in $\mathrm{W}$ ismut krystallisieren zu lassen, wurde in beiden Fällen ein Filz von kleinen wismuthaltigen Nädelchen erhalten, welche in ihrem Äufseren der später noch zu beschreibenden Wismutpalladiumverbindung glichen.

\section{Arsen-, Antimon- und Wismutverbindungen.}

Bei der Verarbeitung der platinhaltigen Rückstände aus der Goldscheidung wird, wie schon erwähnt, zuerst das Platin aus der Lösung in Königswasser durch Salmiak zum allergrölsten Teil ausgefällt und dann das Palladium aus der Mutterlauge als Palladosamminchlorid gewonnen. Es wird durch Ammoniak ein fleischfarbener Niederschlag erzeugt, derselbe in der Hitze im Überschuls des Fällungsmittels wieder gelöst und schliefslich durch Salzsäure dis Palladosamminchlorid als gelbes Salz ausgefällt. Ist der Niederschlag, welcher durch Ammoniak entsteht, rein, so löst er sich beim Erwärmen im Überschufs wieder vollkommen auf. Oft aber fallen mit demselben ein grofser Teil der in der Lösung enthaltenen Verunreinigungen, meist Arsen, Antimon und Wismut, aus, die sich nicht wieder lösen. Sie bilden einen braunen Schlamm, welcher auch beträchtliche Mengen von Platinmetallen zurïckhält. Derselbe, mit Flufs und Kohle geschmolzen, hinterlälst beim Behandehn mit kaltem Königswasser häufig krystallisierte Körperchen. Die Muttermasse besteht gewöhnlich zum grofsen Teil aus Wismut, ist also leicht schmelzbar und bietet daher schwerer schmelzbaren Verbindungen Gelegenheit, sich in Krystallen abzuscheiden. Solche Kryställchen, aus früherer Zeit stammend, enthielten nach der qualitativen Prüfung Platin, Palladium, Kupfer, Wismut, Arsen und Antimon. Unter dem Mikroskop zeigten sich neben gut ausgebildeten Würfeln Gebilde unregelmälsiger Gestalt. Die Körperchen waren also nicht einheitlich und wurde daher eine quantitative Ana- 
lyse nicht unternommen. Einmal enthielt ein König, erhalten beim Zusammenschmelzen des erwähnten Ammoniakniederschlags, eine Druse mit prachtvoll ausgebildeten Krystallen, meist rerzerrten Würfeln (Fig. 21). Einzelne derselbeu zeigten Würfel mit abgestumpften Ecken und Kanten, entsprechend scheinbar einer Kombination von Würfel, Oktaëder und Rhombendodekaëder. Dieselbe ist am Speiskobalt beobachtet worden, welcher, wie die Analyse ergab, eine diesen Krystallen analoge Zusammensetzung hat. Einzelne Beobachtungen deuteten aber darauf hin, dafs hier eine pyritoëdrische Hemiëdrie vorliege, und nicht das Rhombendodekaëder, sondern das Pentagondodekaëder hier die Kanten abstumpfe, entsprechend der in Fig. 22 gezeichneten Kombination. So zeigte eine

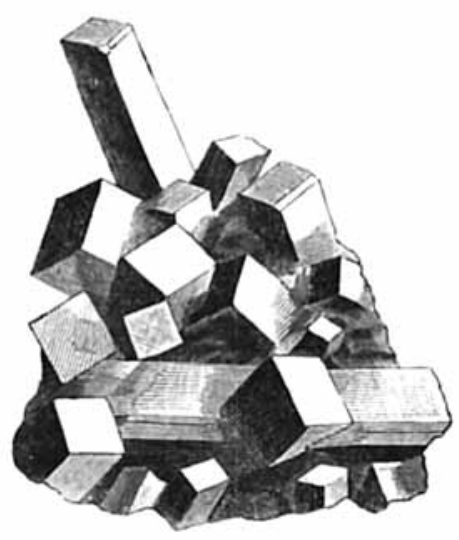

Fig. 21.

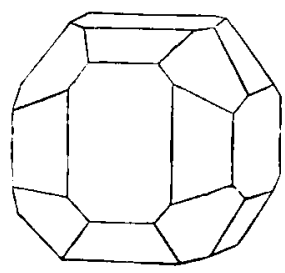

Fig. 22.

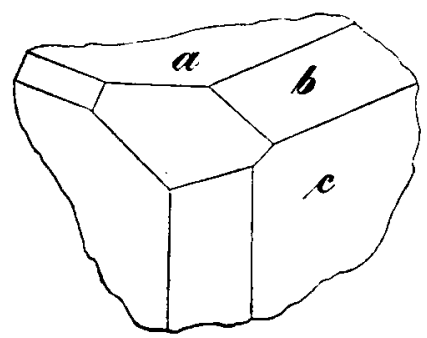

Fig. 23

Würfelecke an den Abstumpfungen deutlich die schiefen Kombinationskanten zwischen $\mathrm{O}$ und $\frac{\infty \mathrm{O} m}{2}$. Dieselbe erlaubte auch eine Messung der Kantenwinkel zwischen $\infty \mathrm{O} \infty$ und $\frac{\infty 0 \mathrm{~m}}{2}$, a. h. in beistehender Figur zwischen $a$ und $b$ und zwischen $b$ und $c$ (Fig. 23). Dieselbe ergab:

$$
\begin{aligned}
& \nless a: b=153^{\circ} 15^{\prime} 50^{\prime \prime} \\
& \nless b: c=116^{\circ} 42^{\prime} 24^{\prime \prime} .
\end{aligned}
$$

Aus $\Varangle a: b=153^{\circ} 15^{\prime} 50^{\prime \prime}$ berechnet sich das Axenverhältnis 
$=1: 0.5037$. Die abstumpfenden Flächen gehören also einer Form $\frac{\infty \mathrm{O} 2}{2}$ an.

Die Krystalle waren hart und spröde. Vor dem Lötrohr entwich viel Arsen, der Rest schmolz zum Korn, welches viel Platin neben etwas Palladium enthielt. Eine trockene Probe durch Ansieden und Abtreiben ergab ca. $45 \%$ Platinmetall. Die quantitative Analyse wurde durch Aufschliefsen im Chlorstrom ausgeführt.

In einem Rohr von schwer schmelzbarem Glase wurde die feingepulverte Substanz im Porzellanschiffchen erhitzt, während ein langsamer Strom trockenen, luftfreien Chlors aus einer Chlorbombe darüber geleitet wurde. Die übergehenden Chloride wurden in zwei hinter einander gelegten, mit Wasser gekühlten VoLHARDschen Torlagen aufgefangen. Dieselben enthielten eime Lösung von 4 Teilen verdünnter Salzsäure $\left(1 \mathrm{HCl}: 4 \mathrm{H}_{2} \mathrm{O}\right)$ und 1 Teil $10 \%$ iger Weinsäure. Die Erhitzung wurde fortgesetzt, bis alle leichter flüchtigen Chloride übergegangen waren und ein dunkelbraunes Sublimat sich in dem kälteren Teile des Rohres ansetzte. Nach dem Erkalten im Chlorstrom wurde das Schiffchen vorsichtig aus dem Rohr genommen und das noch in dem Rohre sitzende Sublimat zu der Lösung in den Vorlagen gespült.

Aus dieser Lösung wurde zunächst durch Aufkochen das Chlor vertrieben und dann längere Zeit unter Erwärmen Schwefelwasserstoff eingeleitet. Der Niederschlag wurde 24 Stunden absitzen lassen, filtriert und das Filtrat nochmals durch längeres Einleiten von Schwefelwasserstoff auf Arsen geprüft. Im Filtrat war dann nur noch etwas bei der Aufschliefsung mit übergegangenes Eisen zu bestimmen. Der Schwefelwasserstoffniederschlag wurde in Königswasser gelöst, ungelöster Schwefel abfiltriert und nach Zusatz von Weinsäure das Arsen aus der möglichst wenig verdünnten Lösung als arsensaure Ammoniak-Magnesia gefällt. Der Niederschlag wurde auf einem bei $100^{\circ}$ getrockneten Filter gewogen. In das Filtrat wurde nun wieder längere Zeit Schwefelwasserstoff eingeleitet. Der geringe Niederschlag bestand hauptsächlich aus Antimonsulfid. Dieses wurde mit frischem Schwefelammonium vom Filter in einen tarierten Tiegel gelöst, eingedampft, mit rauchender Salpetersäure oxydiert und nach dem Glühen als $\mathrm{Sb}_{2} \mathrm{O}_{4}$ ausgewogen. Das Filter, auf dem ein geringer, in Schwefelammonium unlöslicher Rest des Schwefelwasserstoffniederschlages geblieben, wurde verascht und gemeinsam mit 
dem bei der Aufschliefsung in dem Schiffchen gebliebenen Rückstand weiter verarbeitet.

Dieser Rückstand wurde in wenig Königswasser mit der gerade eben genügenden Menge Salpetersäure gelöst, und nach nochmaligem Einengen mit Salzsäure und Wasser das Platin durch festen Salmiak gefällt. Dann wurde in der schon beschriebenen Weise das Palladium durch Aufkochen mit Salpetersäure und Salmiak als Palladiumsalmiak abgeschieden. Nun wurde in die fast farblose, verdümnte Lösung längere Zeit Schwefelwasserstoff unter Erwärmen eingeleitet. Im Filtrat war nur noch Eisen zu bestimmen. Der Schwefelwasserstoffniederschlag wurde mit frischem Schwefelkalium behandelt, um zu priifen, ob etwa Antimon bei der Aufschlielsung nicht mit übergegangen war. Dann wurde der Niederschlag in Salpetersäure gelöst. Dabei blieb ein dunkler, ungelöster Rückstand, welcher sich nach dem Glühen als Gold herausstellte. Aus der verdünnten Lösung wurde wenig Silber, welches zu Anfang in dem konz. Königswasser gelöst geblieben war, mit Salzsäure gefällt. Dann wurde die Lösung mit Schwefelsäure abgeraucht, um die Salzsäure wieder zu entfernen, und aus der schwefelsauren Lauge das Kupfer elektrolytisch gefällt. Wismut, welches sich bei einer früheren Analyse von nicht genau demselben Material gefunden hatte, war in dem zuletzt analysierten Material nicht enthalten. Damals wurde dasselbe nach dem Silber durch Verdünnen gefällt.

Die Analyse ergab:

$$
\begin{aligned}
& \text { In } 0.5164 \mathrm{~g} \text { Material: } \\
& 0.2312 \mathrm{~g} \mathrm{Pt}=44.77 \% \mathrm{Pt} \\
& 0.0128 \mathrm{~g} \mathrm{Pd}=2.48 \% \mathrm{Pd} \\
& 00135 \mathrm{~g} \mathrm{Au}=2.61 \% \mathrm{Au} \\
& 0.0407 \mathrm{~g} \mathrm{Cu}=8.18 \% \mathrm{Cu} \\
& 0.0123 \mathrm{~g} \mathrm{Fe}_{2} \mathrm{O}_{3}=1.67 \% \mathrm{Fe} \\
& 0.0072 \mathrm{~g} \mathrm{AgCl}=1.09 \% \mathrm{Ag} \\
& \text { Summe der Metalle }=60.80^{\circ} \% \text {. } \\
& \text { In } 0.9388 \mathrm{~g} \text { desselben Materials: } \\
& 0.8785 \mathrm{~g} \mathrm{MgNH}_{4} \mathrm{AsO}_{4}+\mathrm{aq}=36.85 \% \mathrm{As} \\
& 0.0128 \mathrm{~g} \mathrm{Sb}_{2} \mathrm{O}_{4}(\operatorname{aus} 0.4975 \mathrm{~g})=2.03 \% \mathrm{Sb}
\end{aligned}
$$

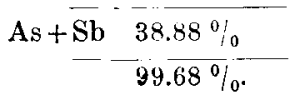

Die Analyse giebt direkt kein einer Formel entsprechendes Resultat. Die Formel PtAs $s_{2}$ verlangt 56.32\% Pt und 43.68\% As. Nun stehen allerdings $\mathrm{Pt}+\mathrm{Au}+\mathrm{Pd}(49.86 \%) \mathrm{zu} \mathrm{Sb}+\mathrm{As}(38.88 \%)$ 
nahezu in demselben Verhältnis. Erwägt man ferner, dals aufgewachsene Krystalle, wie die vorliegenden es waren, meist nicht ganz rein sind, dafs sie Einschlüsse beherbergen, oder Teile der Masse, die als Unterlage dient, ihnen anhaften können, wie man bei dieser Analyse nicht wissen kann, ob Kupfer und Eisen aus solchen Verunreinigungen stammen oder an der Konstitution teilnehmen; endlich, dafis gerade der den Krystallen offenbar isomorphe Speiskobalt oft infolge solcher Beimengungen bei der Analyse einen zu geringen Arsengehalt angiebt, ${ }^{1}$ so kann man die Zusammensetzung nach der Formel $\mathrm{PtAs}_{2}$ im Grolsen und Ganzen als erwiesen annehmen. Für die Richtigkeit dieser Annahme spricht aber besonders der folgende Versuch.

Es wurde nämlich versucht, die zuerst erwähnten, beim $\mathrm{Zu}$ sammenschmelzen des braunen Schlammes in der gesschmolzenen Masse auskrystallisierten Körperchen, die ja ihrer Entstehung nach eine ähnliche Zusammensetzung wie die untersuchten Krystalle erwarten liefsen, nochmals, eventuell reiner und grölser zu erhalten. Es wurde zu diesem Zwecke eine gröfsere Menge des braunen Ammoniakniederschlags gesammelt, getrocknet und mit Fluls und Kohle geschmolzen. Der Versuch gelang über Erwarten gut. Es erfolgte ein König, welcher in einer Muttermasse, die aus viel Wismut und Kupfer neben Platinmetallen bestand, prachtvolle Krystalle enthielt, welche sich in kaltem Königswasser unversehrt auslösen liefsen.

Die Krystalle waren im Gegensatz zu den in einer Druse gebildeten Krystallen aus einer leichter schmelzbaren Muttermasse frei heraus krystallisiert. Sie waren dabei in der noch flüssigen Masse zu Boden gesunken und salsen daher meist an der unteren Seite des Königs. Sie waren daher auch reiner und genauer der Formel PtAs $\mathrm{s}_{2}$ entsprechend zusammengesetzt, als jene aufgewachsenen Krystalle, wie die untenstehende Analyse ergab.

Was ihre Form anbetrifft, so bildeten sie Gruppen von teils treppenförmig, teils unregelmälsig durch einander gewachsenen Würfeln, darunter einige mit abgestumpfen Ecken. Eine Abstumpfung. der Kanten wurde nicht beobachtet. Die Krystalle waren hübsch ausgebildet und hatten die meisten Durchmesser von mehreren Millimetern (Fig. 24). Oft salsen auf den treppenförmigen Gruppen noch kleine Würfelchen. Sie zeigten eine unvollkommene Spaltbarkeit nach dem Würfel. Die Oberfläche der Krystalle war

${ }^{1}$ Tschermak, Mineralogie, S. 338. 
rauh, die Farbe bleigrau, auf den Spalttlachen metallglänzend. Auffallend war die grolse Härte, die etwa der des Quarzes gleichkam.

Die Analyse wurde in derselben Weise wie bei den anderen Krystallen ausgeführt. Sehr interessant war ein Gehalt an Iridium. Der grölste 'Teil desselben blieb beim Auflösen des Rückstandes aus dem Schiffchen ungelöst. Geringe Mengen, die beim Kochen mit dem konz. Königswasser doch in Lösung gegangen waren, fanden sich nach dem Ausfällen des Platins und Palladiums im
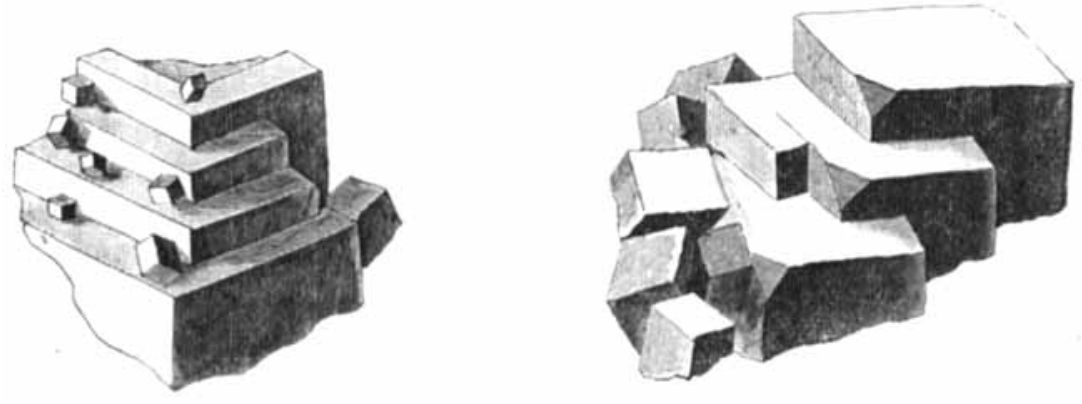

Fig. 24.

Schwefelwasserstofiniederschlag. Auch enthielten die Krystalle etwas $\mathrm{Bi}$, dagegen kein $\mathrm{Au}$ und $\mathrm{Ag}$. Die Analyse ergab in $0.8275 \mathrm{~g}$

$$
\begin{aligned}
& 0.4225 \mathrm{~g} \mathrm{Pt} \quad=51.07 \% \mathrm{Pt} \\
& \text { Spur Pd } \\
& 0.0342 \mathrm{~g} \mathrm{I} r \\
& 0.0055 \mathrm{~g} \mathrm{Cu} \text { (ans } 1.0455 \mathrm{~g} \text { ) }=0.42 \% \mathrm{Cu} \\
& 0.0037 \mathrm{~g} \mathrm{Fe}_{2} \mathrm{O}_{3} \quad=0.25 \% \mathrm{Fe} \\
& \text { Summe der Metalle } 56.00 \% \\
& 0.8340 \mathrm{~g}^{\mathrm{MgNH}} \mathrm{Is}_{4}+\mathrm{Aq}=39.69{ }^{\circ}{ }_{0} \mathrm{As} \\
& 0.0230 \mathrm{y} \mathrm{Sb}_{2} \mathrm{O}_{4}=2.19 \% \mathrm{Sb} \\
& 00088 \mathrm{~g} \mathrm{Bi}_{2} \mathrm{O}_{3} \quad=0.95 \% \mathrm{Bi} \\
& \mathrm{As}+\mathrm{Sb}+\mathrm{Bi}=\frac{42.83 \%}{98.83 \%}
\end{aligned}
$$

Die Krystalle entsprechen also ziemlich genau der Formel $\mathrm{PtAs}_{2}$, welche $56.32 \%$ Pt und $43.68 \%$ As verlangt.

Nachlem ich meine Untersuchungen über diese künstlichen Arsenplatinkrystalle schon beendet batte, wurde mir durch die Güte des Herm Geheimrat Cr. Winkler in Freiberg eine Abhandlung 
zur Verfügung gestelit, welche ein natürliches Arsenplatinvorkommen, den Sperrylith, behandelt. ${ }^{1}$ Derselbe ist in den meisten neueren Handbüchern noch nicht erwähnt. Dieser Sperrylith stimmt in jeder Beziehung mit den hier untersuchteu künstlichen Krystallen überein. Seine $\mathrm{Zu} \backsim a m m e n s e t z u n g$ ist $\mathrm{PtAs}_{2}$ mit etwas Rhodium und etwas Antimon. Seine Krystallform ist regulär, meist der Würfel in Kombination mit dem Oktaëder. Auch die Abstufung cler Würfelkanten durch das Pentagondodekaëder $\stackrel{\infty 02}{2} \frac{2}{2}$ wurde am natürlichen Arsenplatin beobachtet. Seine Farbe, die unvollkommene Spaltbarkeit, seine Härte, welche als zwischen der des Feldspats und der des Quarzes stehend beobachtet wurde, stimmen ebenfalls mit den Eigenschaften der künstlichen Krystalle überein.

Nachdem festgestellt war, dals sich in einer geschmolzenen Muttermasse Krystalle von der Zusammensetzung $\mathrm{PtAs}_{2}$ gebildet hatten, hätte ja die synthetische Darstellung von krystallisierten Arsenverbindungen des Platins unl Palladiums am nächsten gelegen. Dafs beim Zusammenschmelzen von Arsen mit Platin oder Palladium die Verbindungen $\mathrm{PtAs}_{2}$ und $\mathrm{PdAs} \mathrm{s}_{2}$ als stahlgraue. spröde. amorphe Körper entstehen, ist bekannt. ${ }^{2}$ Un aber Krystalle durch Lösen in einern Schmelzflufs zu erzielen, hätte man entweder, da ja Arsen sich nicht in offenen Gefäfsen einschmelzen lälst, ein Verfahren einschlagen müssen, wie MARGотTET es zur Darstellung krystatlisierten Silberglanzes im geschmolzenen Schwefel verwendete, indem er denselben im geschlossenen Rohr erhitzte, oder man hätte, analog dem Vorgang bei der zufälligen Bildung der Arsenplatinkrystalle, eine indifferente, leichter schmelzbare Legierung als Lösungsmittel anwenden müssen. Ein solches wäre aber erst durch eine Reihe von Versuchen aufzufinden gewesen. Es erschien daher einfacher, den Arsenverbindungen analoge Antimon- und Wismutverbindungen darzustellen, da sich dabei Wismut und Antimon selbst als leichter schmelzbares Krystallisationsmittel benutzen lielsen.

\section{Antimonplatin.}

Nach Sartorius v. Waltershausen ${ }^{3}$ verbinden sich 1 Teil Platinschwamm und 2 Teile Antimonpulver leicht unter Èrglühen.

1 Amer. Journ. Se. [Sill.] (1889) Vol. XXXll. Sperrylithe a new minerale by Morace: L. WeLIS.

2 GMELIN-KRACT, 2, 1192.

${ }^{3}$ Gmalin-Kratt, 2, 1192. 
Beim Schmelzen in der Rotglut hinterbleilst ein spröder; grauer Regulus mit krystallinischem Bruch. In den Höhlungen soleher Könige fand Christoffre schöne hexagonale Krystalle. ${ }^{1}$

Nachdem eine vorläufige Schmelzung von $28 \mathrm{~g}$ Antimon und $0.5 \mathrm{~g}$ Platin die Bildung von hübschen Krystallen erwiesen hatte, wurde ein grölserer Versuch gemacht. Es wurden $400 \mathrm{~g}$ gepulvertes Antimon mit $10 \mathrm{~g}$ Platinpulver unter einer Glasdecke im Koksofen zusammengeschmolzen. Der erhaltene König zeigte auf der Bruchfläche einige Drusen, in welchen einige würfelähnliche Krystalle safsen. Das Antimon besals seine gewöhnliche Struktur, vielleicht etwas feiner krystallinisch, wie reines Antimon. Auch zeigten sich hellere Pünktchen in demselben. Zur Auflösung der Kryställchen wurde hier, wie bei den folgenden Antimonverbindungen, eine Mischung von 1 Teil verdünnter Salpetersäure $(1.1$ spez. Gew.) und 1 Teil konz. Weinsäurelösung angewendet. Nach längerer Einwirkung der Säure zeigten sich in dem König hübsche Kryställchen, welche fast nur an der unteren Aufsenseite des Königs safsen und dort ein hübsches Relief bildeten. Auch hier waren also die schwereren Krystalle in der noch flüssigen Muttermasse zu Boden gesunken und dort weitergewachsen, gerade wie Kochsalzkrystalle beim Verdunsten einer gesättigten Lösung. Es lielsen sich hübsche, zum Teil mehrere Millimeter grolse Kryställchen, oft zu mehreren verwachsen, herauslösen. Sie waren dunkelgrau, mit glänzenden, oft eingefallenen Flächen, sehr hart und so spröde, dals sich nur wenige ringsum unversehrte Krystalle isolieren liesfen. Mehrere derselben zeigten deutlich die Kombination von Würfel und Oktaëder, mit vorwaltendem Oktaëder (Fig. 25), meistens jedoch in sehr einseitiger Ausbildung, so dals sich viele Krystalle von ähnlicher Form, wie nachstehende Zeichnung (Fig. 26), vorfanden.

Die Analyse wurde durch Aufschliefsen im Chlorstrom ausgeführt. Sie ergab die Formel $\mathrm{PtSb}_{2}$. Es fanden sich in $0.4140 \mathrm{~g}$ $0.1837 \mathrm{~g} \mathrm{Pt}$, in $0.5394 \mathrm{~g} 0.4068 \mathrm{~g} \mathrm{Sb}_{2} \mathrm{~S}_{3}+0.0073 \mathrm{~g} \mathrm{Sb}_{2} \mathrm{O}_{4}$.

\begin{tabular}{l} 
Gefunden: \\
$44.37 \% \mathrm{Pt}$ \\
$54.93 \% \mathrm{Sb}$ \\
\hline $99.30 \%$
\end{tabular}

Berechnet: $44.83 \% \mathrm{Pd}$ $55.17 \% \mathrm{Sb}$. $100.00 \%$

1 Christoffre, Recherches sur les combinaisons de l'antimoine (Göttingen 1863 \% 


\section{Wismutplatin.}

Nach GeHLen ${ }^{1}$ verbinden sich 1 Teil Platinschwamm und 2 Teile Wismut leicht obne Feuererscheinung zu einer spröden leichtflüssigen Masse von blätterigem Bruch.

Nachdem ein vorläufiger Versuch die Bildung von Krystallen erwiesen hatte, wurden $400 \mathrm{~g}$ Wismut mit $10 \mathrm{~g}$ Platin unter Glas im Koksofen zusammengeschmolzen und langsam erkalten lassen. Beim Behandeln des Königs mit verdünnter kalter Salpetersäure zeigte sich, dals auch hier die grofse Masse der Kryställchen an

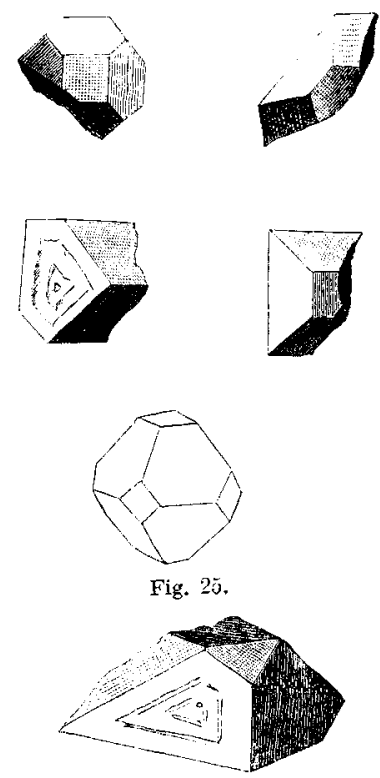

Fig. 26.
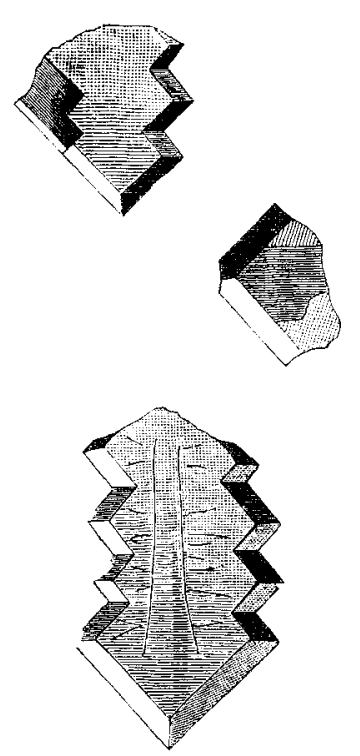

Fig. $2 \overline{7}$.

der Aufsenseite des Königs salsen. Doch war auch das Innere desselben von kleinen Kryställchen durchsetzt. Es waren dunkelgraue, braun und blau anlaufende, aufserordentlich spröde, unregelmälsig durcheinander gewachsene Blättchen, zum Teil von einigen Millimetern Durchmesser. Sie zerbrachen sehr leicht und zeigten daher nur wenige die nachstehenden, wohl quadratischen Formen unter dem Mikroskop (Fig. 27).

Zum Zweck der Analyse wurde das Material in möglichst wenig Königswasser gelöst, indem zu wenig konz. Salzsäure nur gerade

1 Gmelin-Kraut 2, 1093. 
so viel Salpetersäure tropfenweise zugegeben wurde, als zum Lösen des Materials erforderlich war. Aus der Lösung wurde zuerst Wismut durch Verdünnen als $\mathrm{BiOCl}$ gefällt und auf einem gewogenen Filter getrocknet und gewogen. Dann wurde die Lösung durch Eindampfen möglichst eingeengt, das Platin als Platinsalmiak gefällt und nach dem Glühen als Metall gewogen. Ein wenig Wismut, das noch in Lösung geblieben, wurde zuletzt durch Schwefelwasserstoff ausgefällt, mit dem Filter verascht, mit Salpetersäure oxydiert, geglüht und als $\mathrm{Bi}_{2} \mathrm{O}_{3}$ ausgewogen. Sonderbarerweise ergab die Analyse auch noch $0.85 \%$ Silber, welches vor dem Fällen des Wismuts als Chlorsilber abfiltriert wurde. Das mir zur Verfügung stehende Wismut gilt als technisch rein. Jedoch kann zuweilen ein Silbergehalt bis zu höchstens $0.1 \%$ in demselben vorkommen. Es hat sich hier also offenbar der geringe Silbergehalt des Wismuts in der Platinwismutverbindung angesammelt. Das Resultat der Analyse weicht etwas von der erwarteten Formel $\mathrm{PtBi}_{2}$ ab, was jedenfalls darauf zurückzuführen ist, dafs durch die Einwirkung auch der verdünnten Säure sich leicht etwas Wismut aus der Verbindung auslöst, ohne dafs Platin mit in die Lösung gehen kann.

Es fanden sich in $1.1047 \mathrm{~g}$ Material $0.3765 \mathrm{~g} \mathrm{Pt}, 0.8757 \mathrm{~g}$ $\mathrm{BiOCl}+0.0063 \mathrm{~g} \mathrm{Bi}_{2} \mathrm{O}_{3}$ und $0.0125 \mathrm{~g} \mathrm{AgCl}$.

\begin{tabular}{cc} 
Gefunden: & Berechnet: \\
$34.08 \% \mathrm{Pt}$ & $31.92 \% \mathrm{Pt}$ \\
$64.19 \% \mathrm{Bi}$ & $68.08 \% \mathrm{Bi}$ \\
$0.85 \% \mathrm{Ag}$ & - \\
\hline $99.12 \%$ & $100.00 \%$
\end{tabular}

Antimonpalladium.

Palladium und Antimon verbinden sich nach Fischer ${ }^{1}$ unter Feuererscheinung zu einer schmelzbaren spröden Verbindung.

$20 \mathrm{~g}$ Antimon und $0.5 \mathrm{~g}$ Palladium wurden unter Glaspulver zusammengeschmolzen und langsam erkalten lassen. Beim Auflösen des erhaltenen Köönigs in Salpeter-Weinsäure hinterblieb ein Pulver kleiner grauer Körperchen. Dieselben machten zwar den Eindruck von Krystallen oder Krystallsplittern, waren aber offenbar von der Säure angefressen und liefsen keine bestimmte Krystallform erkennen. Einige zeigten eine federartige Anordnung. Auch hier ergab die Analyse nur annähernd die erwartete Zusammensetzung $\mathrm{PdSb}_{2}$.

1 Grelin-Kraut $2,1252$. 
Eine trockene Probe ergab $33 \% \mathrm{Pd}$. Eine Analyse auf nassem Wege ergab in $0.6715 \mathrm{~g} 0.2326 \mathrm{~g} \mathrm{Pd}$.

$$
\begin{array}{cc}
\text { Gefunden: } & \text { Berechnet: } \\
34.64 \%_{0}^{\circ} \mathrm{Pd} & 30.74^{\circ} \%_{\mathrm{h}} \mathrm{Pd} .
\end{array}
$$

Auch hier muls man wohl eine Einwirkung der Säure auf die Kryställchen annehmen, die bei der Kleinheit der Körperchen naturgemäl's viel stärker sein mul's. Eine gröl'sere Schmelzung wurde nicht unternommen, da kein besseres Resultat zu erwarten war.

Wismutpalladium.

Palladium und Wismut zu gleichen Teilen zusammengeschmolzen geben eine graue, spröde, stahlharte Legierung. ${ }^{1}$

Es wurden $20 \mathrm{~g}$ Wismut und $1 \mathrm{~g}$ Palladium zusatmmengeschmolzen. Der erhaltene König war auffallend spröde. Beim Behandeln mit verdünnter Salpetersäure zeigten sich in demselben kleine Nädelchen, und bei weiterer Einwirkung der Säure hinterblieb zuletzt eine filzige Masse, aus kleinen, zum Teil nur mikroskopisch erkennbaren Nädelchen bestehend. Bestimmbare Formen liefsen sich an den Kryställchen, die meist büschelförınig zusammengewachsen waren, nicht erkennen. Die Analyse wurde ebenso ausgeführt wie die der Wismutplatinverbindung and ergab die Formel $\mathrm{Pd} \mathrm{Bi}_{2}$. In 0.7620 $\mathrm{g}$ fanden sich 0.7528 $\mathrm{g} \mathrm{BiOCl}$ und $0.1582 \mathrm{~g} \mathrm{~Pa}$.

\begin{tabular}{cc} 
Gefunden: & Berechnet: \\
$20.76 \% \mathrm{Pd}$ & $20.31 \% \mathrm{Pd}$ \\
$79.14 \% \mathrm{Bi}$ & $79.69 \% \mathrm{Bi}$ \\
\hline $99.90 \%$ & $100.00 \%$
\end{tabular}

Die hier beschriebenen Arsen-, Antimon und Wismutrerbindungen des Platins und Palladiums sind also nach dem Typus des Pyrits zusammengesetzt. In der allgemeinen Formel $\mathrm{MeX}_{2}$ treten hier für Me Pt und $\mathrm{Pl}$, für $\mathrm{X} \mathrm{As}$, Sb und $\mathrm{Bi}$ ein. Arsenplatin und Antimonplatin zeigen auch die Formen des regulären Systems, welche vielen Mineralien jener Gruppe eigentümlich sind.

\section{Wismutgold.}

Beim Zusammenschmelzen von $100 \mathrm{~g}$ Wismut und $3 \mathrm{~g}$ Gold wurde ein Wismutkönig erhalten, der beim Auflösen in rerdünnter

1 Gielix-Kriati 2, 1252. 
kalter Salpetersäure (1.1 spez. Gew.) ein gelbgranes Krystallpulver hinterliels. Dasselbe enthält nach einer Abtreibeprobe ca. $74 \% \mathrm{Au}$. Hier wurde nun eine Anzahl Versuche angestellt, um grölsere Kryställchen zu erzielen.

Zunächst wurde Wismut in einem offenen Thonschälchen über einem gewöhnlichen Brenner geschmolzen. Es löste, nur wenig über seinen Schmelzpunkt erhitzt, ein hineingehängtes Goldstreifchen rasch und vollständig auf. Das Wismut wurde mehrere Stunden lang im Hïssigen Zustand erhalten und dann langsam erkalten lassen.

Ferner wurde Wismut mit etwa $3 \%$ Gold in einem Luftbade bei $300^{\circ}$ längere Zeit flüssig gehalten, darauf bei langsam sinkender Temperatur (bis $250^{\circ}$ ) erstarren lassen, dann wieder längere Zeit bis $300^{\circ}$ erhitzt, wieder erstarren lassen und dieser Vorgang mehrere Male wiederholt. Diese Behandlungsweise wurde zuerst von Satnte-Craire Devilite ${ }^{1}$ angewendet, um schwerlösliche Substanzen in gröfseren Krystallen zu erhalten.

Endlich wurde noch eine gröfsere Schmelzung von $400 \mathrm{~g}$ Wismut und $10 \mathrm{~g}$ Gold ziemlich heifs gemacht und im Ofen sehr langsam erkalten lassen. Bei allen diesen Versuchen wurden keine erheblich gröfseren Krystalle erhalten.

Auf der frischen Bruchfläche zeigten die Wismutkönige auffallend kleinere Krystallbildung als reines Wismut und auf den Krystallfächen helle Pünktchen. Beim Behandeln mit verdünnter Salpetersäure erwies sich der ganze König mit kleinen teils grauen, teils messingfarbenen Kryställchen durchsetzt. Unter dem Mikroskop zeigten sie sich meist

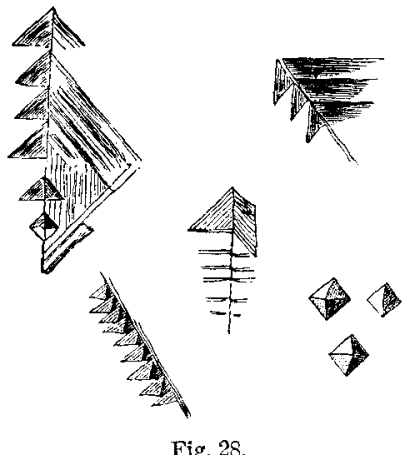

Fig. 28. zu sonderbaren Gruppen vereinigt. Einzelne hatten eine federartige Anordnung. Auch liefsen sich einige kleine Oktaëder erkennen (Fig. 28). Die verschiedene Färbung schien nur auf Kinwirkung der Säure zurückzuführen und die Kryställchen trotzdem einheitlich $\mathrm{zu}$ sein.

Bei der Analyse fand sich wieder ein Silbergehalt der Krystalle, welcher hier, auf die Menge des rerwendeten Wismuts berechnet, etwa $1 / 1000$ derselben betrug, so dals man auch hier annehmen kann,

1 Dowtren, Chemische Mineralogie, S. 123. 
dafs der geringe Silbergehalt des Wismuts sich in den Kryställchen angereichert hat. Bei der Aufstellung einer Formel muls der Silbergehalt jedenfalls zum Gold gerechnet werden. Dann stimmt die Zusammensetzung ziemlich gut mit der Formel $\mathrm{Au}_{3} \mathrm{Bi}$. Es fanden sich in $0.3230 \mathrm{~g} 0.2277 \mathrm{~g} \mathrm{Au}, 0.0115 \mathrm{~g} \mathrm{AgCl}, 0.0957 \mathrm{~g} \mathrm{BiOCl}$.

\begin{tabular}{cc} 
Gefunden: & Berechnet: \\
$\left.\begin{array}{rl}70.49 \% \mathrm{Au} \\
2.68 \% \mathrm{Ag}\end{array}\right\} 73.17 \%$ & $73.97 \% \mathrm{Au}$ \\
$26.56 \% \mathrm{Bi}$ & $26.03, \mathrm{Bi}$ \\
\hdashline $99.75 \%$ & $100.00 \%$
\end{tabular}

Eine trockene Probe ergab $74.20 \% \mathrm{Ag}+\mathrm{Au}$.

Antimongold.

$100 \mathrm{~g}$ Antimon mit $3 \mathrm{~g}$ Gold ergaben einen König, welcher auf der frischen Bruchtläche sich von reinem Antimon höchstens durch etwas kleinere Krystallbildung unterschied. Nach kurzer Einwirkung der Säure zeigten sich in dem König klcine bronzerote Körperchen, deren Menge nach aulsen zunahn und welche anf der Aufsenseite des Königs zu grölseren Anhäufungen rereinigt waren, so dafs sie stellenweise ein deutliches Relief auf derselben bildeten. Unter dem Mikroskop machten einige der Körperchen wohl den Eindruck einer krystallisierten Substanz, liefsen aber keine bestimmbaren Formen erkennen. Andere schienen stark ron der Säure angegriffen. Auch Schmelzungen mit $10 \%$ und mehr Gold ergaben keine grölseren Krystalle. Die rote Farbe war wohl nur auf Einwirkung der Säure zurïckzuführen. Denn eime Legierung ron $20 \%$ Antimon und $80 \%$ Gold, ungefähr der Formel $\mathrm{Au}_{3} \mathrm{sb}$ entsprechend, zeigte auf der frischen Bruchtäche eine graue, auf der mit Salpeterweinsäure behandelten Fläche eine bronzerote Farbe, wie die Kryställchen. Eine trockene Probe durch Ansieden und Abtreiben ergab in dem zuerst ausgelösten Material $82 \% \mathrm{Au}$. Die Formel $\mathrm{Au}_{3} \mathrm{Sb}$, analog der vorhergehenden Verbindung $\mathrm{Au}_{3} \mathrm{Bi}$, verlangt $83.12^{\circ}{ }_{\circ} \mathrm{Au}$. Die später ausgelösten K̈̈rperchen erschienen unter dem Mikroskop mit weifsen und grauen Partikelchen verunreinigt, die sich nicht völlig herauswaschen liefsen. Sie enthielten daher nur 80.95 ${ }_{0}^{\circ}$ Au. Bei Versuchen, durch weitere Behandlung mit rerdünnter Salpetersäure diese Verunreinigungen herauszulosen, wurden auch die Goldantimonkörperchen angegriffen. Da ilso kein ganz zuverlässiges Material 
zu erhalten war, so kann man eine Zusammensetzung nach der Formel $\mathrm{Au}_{3} \mathrm{Sb}$ nur als wahrscheinlich annehmen.

Es folgen nun noch einige Versuche, die nicht weiter verfolgt wurden, die aber zeigen, welcher Ausdehnung diese Methode zur Darstellung krystallisierter Metallverbindungen fähig ist.

So wurden $2 \mathrm{~g}$ Nickel in $40 \mathrm{~g}$ Wismut eingeschmolzen. Es zeigten sich beim Auflösen kleine, durcheinander gewachsene Nädelchen, welche Nickel und Wismut enthielten. Ähnliche Kryställchen wurden beim Einschmelzen von Nickel in Antimon erhalten.

Speiskobaltkryställchen wurden dadurch dargestellt, dals man gepulverten, natürlichen Speiskobalt unter Glas in geschmolzenem Blei auflöste und wieder auskrystallisieren liefs. Es zeigten sich in dem Bleikönig kleine Drusen mit schönen, zu Gruppen vereinigten Würfelchen, von welchen einige Abstumpfungen der Ecken, andere Zeichnungen auf den Würfelflächen erkennen liefsen. Auch beim Zusammenschmelzen von $3 \mathrm{~g}$ Kobaltoxyd, $6 \mathrm{~g}$ Arsen, $1 \mathrm{~g}$ Kohle und $2 \mathrm{~g}$ Soda mit $100 \mathrm{~g}$ Blei unter Glasdecke wurden dieselben Würfelchen erhalten.

Die in dieser Arbeit beschriebenen Versuche haben bewiesen, dafs man die Fähigkeit der Metalle, im geschmolzenen Zustand gewisse Verbindungen aufzulösen und dieselben beim Erkalten wieder abzuscheiden, sehr gut zur. Synthese krystallisierter, künstlicher Mineralien benutzen kann. Jedoch können, wie die eingehenden Versuche mit Schwefelsilber gelehrt, und alle ausgeführten Synthesen bestätigt haben, die betreffenden Substanzen sich nur dann in Krystallen abscheiden, wenn sie schwerer schmelzen als die Metalle, welche sie gelöst enthalten.

Folgende Tabelle enthält die im Laufe dieser Arbeit beschriebenen Verbindungen. 


\begin{tabular}{|c|c|c|c|}
\hline Bezcichnung & Formel & Krystallform & Min. Name \\
\hline $\begin{array}{l}\text { Schwefelblei } \\
\text { Selenblei } \\
\text { Schwefelwismut } \\
\text { Selenwismut } \\
\text { Selensilber } \\
\text { Schwefelsilberwismut } \\
\text { Selensilberwismut }\end{array}$ & $\begin{array}{c}\mathrm{PbS} \\
\mathrm{PbSe} \\
\mathrm{Bi}_{2} \mathrm{~S}_{3} \\
\mathrm{Bi}_{2} \mathrm{Se}(?) \\
\mathrm{Ag}_{2} \mathrm{Se} \\
\mathrm{AgBiS}_{2} \\
(\mathrm{Ag} \cdot \mathrm{Bi})_{2} \mathrm{Se}(?)\end{array}$ & $\begin{array}{c}\text { Reguläre Würfel } \\
\text { " " } \\
\text { Rhombische Nädelchen } \\
\text { Reguläre Oktaëder } \\
\text { Reg. Rhombendodekaëder } \\
\text { Reguläre Oktaëder } \\
, ", "\end{array}$ & $\begin{array}{c}\text { Galenit } \\
\text { Clausthalit } \\
\text { Bismutit } \\
\text { Naumannit }\end{array}$ \\
\hline Arsenplatin & $\mathrm{PtAs}$ & Regulär. $\infty 0 \infty \cdot \frac{0 . \frac{\infty 02}{2}}{\infty}$ & Sperrylith \\
\hline $\begin{array}{l}\text { Antimonplatin } \\
\text { Wismutplatin } \\
\text { Antimonpalladium } \\
\text { Wismutpalladium }\end{array}$ & $\begin{array}{l}\mathrm{PtSb}_{2} \\
\mathrm{PtBi}_{2}(?) \\
\mathrm{PdSb}_{2}(?) \\
\mathrm{PdBi}_{3}\end{array}$ & $\begin{array}{c}\text { Regulär. Oktaëder u. Würfel } \\
\text { Quadratische (?) Blättchen } \\
\text { ? } \\
\text { Nädelchen }\end{array}$ & \\
\hline $\begin{array}{l}\text { Wismutgold } \\
\text { Antimongold }\end{array}$ & $\begin{array}{l}\mathrm{Au}_{3} \mathrm{Bi} \\
\mathrm{Au}_{3} \mathrm{Sb}(?)\end{array}$ & $\underset{?}{\text { Regulär (?) }}$ & \\
\hline
\end{tabular}

In amorphem Zustand dargestellt, analysiert und beschrieben wurden aufserdem:

$$
\begin{aligned}
& \text { Palladiumsubsulfür . . } \quad \text { Pds, } \\
& \text { Palladiumselenür . . : PdSe, } \\
& \text { Platinselenür . . . } \quad \text { PtSe. }
\end{aligned}
$$

Im Laufe der Versuche wurde mehrfach beobachtet, dafs Schwefel und Selen unter ganz gleichen äufseren Verhältnissen keine analogen Verbindungen bildeten.

So ergab Schwefel, in Wismut eingetragen, dem natürlichen Wismutglanz entsprechende Kryställchen, während Selen in Wismut nicht die erwartete, jenen isomorphe Verbindung $\mathrm{Bi}_{2} \mathrm{Se}_{3}$, sondern reguläre Oktaëder, wahrscheinlich von der Formel $\mathrm{Bi}_{2} \mathrm{Se}$ bildete.

Beim Einschmelzen von Schwefelsilber in Wismut entstanden Oktaëder von der Zusammensetzung $\mathrm{AgBiSe}{ }_{2}$, indem sich Wismut gegen silber umsetzte. Selensilber dagegen löste sich in Wismut unverändert auf und krystallisierte beim Erkalten in Rhombendodekaëdern ans.

Während Palladium mit einem Überschuls von Schwefel unter Borax geschmolzen die Verbindung $\mathrm{Pd}_{2} \mathrm{~S}$ ergab, erhielt man beim Schmelzen von Palladium mit überschüssigem Selen einen Körper von der Zusammensetzung PdSe.

Platin und Schwefel lielsen sich nicht zusammenschmelzen, während Platin und Selen unter Borax die Verbindung PtSe ergaben, die erst beim Glühen an der Luft ihr Selen verlor. 
Im überschüssigen Palladium bildete Schwefel Körperchen von der Formel $\mathrm{Pd}_{2} \mathrm{~S}$, während Selen hier Körperchen bildete, welchen wahrscheinlich die Formel $\mathrm{Pd}_{4} \mathrm{Se}$ zukommt.

Platin und Palladium bildeten im geschmolzenen Antimon und Wismut Verbindungen nach dem Typus des Pyrits, wie z. B. $\mathrm{PtSh}_{2}$. Gold bildete im Wismut die Verbindung $\mathrm{Au}_{3} \mathrm{Bi}$ und eine derselben analoge wahrscheinlich auch im Antimon.

\section{Anhang.}

Durch die Lektüre des Werkes von Prof. BenRens' in Delft wurde ich darauf gebracht, auch bei den von mir ausgefïhrten Versuchen den Einflufs der Abscheidung gewisser Verbindungen in den Metallen auf die Struktur derselben etwas zu untersuchen.

Benrens führt in jenem Werke aus, dafs fast jedes Metall krystallinisch erstarre, dals aber bei reinem Metall die Gruppen der Krystalle so vollkommen in einander wachsen, dafs die krystallinische Struktur nicht mehr zu erkennen ist. Scheiden sich jedoch zwischen den Krystallen Oxyde oder Sulfide aus, so können dieselben den Zusammenhang der Krystalle der Metallmasse beeinträchtigen. Hierdurch erklärt sich die ganz allgemein beobachtete Erscheinung, dafs geringe Mengen von Schwefel, Selen, Antimon, Arsen etc. manche Metalle leichtbrüchig machen, indem sie in denselben Verbindungen bilden, wie sie in der vorliegenden Arbeit beschrieben sind.

So zeigte Silber, welches $2 \%$ Schwefelsilber enthielt, auf der polierten und geätzten Schnittfäche unter dem Mikroskop sehr hübsch die Schwefelsilberkörperchen in unregelmälsigen Linien angeordnet, wie sie sich zwischen den Silberkrystallen ausgeschieden haben (Fig. 29). Durch diesen Schwefelsilbergehalt wird das Silber leichter brüchig und hat einen grauen, grofszackigen Bruch. Auch auf der Oberfläche der Silberbarren zeigte sich ein Gehalt an Schwefel oder Selen meistens durch stark hervortretende Krystallbildung an (vergl. Fig. 30). Auf der Oberfläche eines Feinsilberbarrens dagegen kann man meistens beobachten, wie die Silberkrystalle in einander gewachsen sind. Die Oberfläche ist vollkommen glatt und zeigt nur in schräg auffallendem Licht teils matte, teils hell glänzende, zackig in einander greifende Felder. Unter dem

' Bennens, Das mikroskopische Gefüge der Metalle und Legienzmgen. Hamburg und Leipzig, Leop. Voss. S. 2 ff. 
Mikroskop zeigt sich, dals dieser verschiedene Glanz ron einer aufserordentlich feinen Riefung herrührt, welche der Richtung entspricht, in welcher sich die kleinen Silberkryställchen zu gröiseren Gruppen vereinigen, die dann wiederum zu einer einheitlichen Masse zusammenwachsen. Der Bruch solchen reinen Silbers ist weifs und zähe.

Noch schöner lassen sich ähnliche Erscheinungen an Gold be. obachten. Feingoldbarren zeigen oft auf der Oberfläche sehr feine, krystallinische Zeichnungen, die durch die verschiedene Wachstumsrichtung der Krystallgruppen entstehen und in den einzehnen

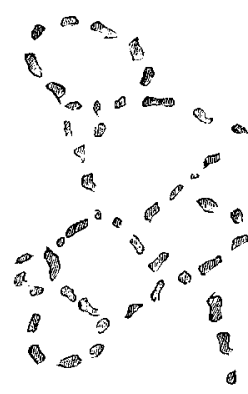

Fig. 29.

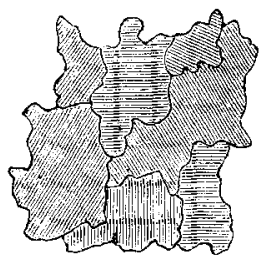

Fig. 30.

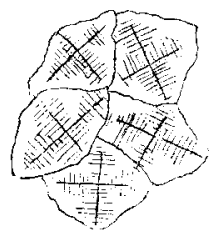

Fig. 31 .

Gruppen zwei senkrecht zu einander stehende Axen erkennen lassen (Fig. 31).

$20 \mathrm{~g}$ chemisch reines Gold wurden nach dem Schmelzen in ein flaches Thonschälchen ausgegossen und das erhaltene, flache Metallstück mit Königswasser geätzt. Weitere $20 \mathrm{~g}$ Gold, denen jedoch $200 \mathrm{mg}=1 \%$ Blei zugesetzt worden war, wurden ebenso bebandelt. Unter dem Mikroskop zeigte das reine Gold eine grofse Zahl dicht aneinander geschlossener, unregelmälsiger Felder von verschiedenem, je nach der Beleuchtung wechselndem Glanz (Fig. 32).

Das bleihaltige Gold dagegen zeigte kleinere Felder, durch dunkle Furchen von einander getrennt. In diesen hatte sich offenbar eine leichter schmelzbare Bleigoldverbindung abgeschieden, welche von der Säure stärker angegriffen wurde, als das reine Gold (Fig. 33). Solches bleihaltiges Gold ist daher sehr spröde und lälst sich leicht durchbrechen, während reines Gold sich bekanntlich durch seine aufserordentliche Duktilität auszeichnet. In der Nähe der Bruchstelle zeigte ein Stïck bleihaltiges Gold Risse, welche genau den Fugen zwischen den einzelnen Krystallen folgten (Fig. 34). Die zwischen den Krystallen ausgeschiedene Bleigoldlegierung lief's sich nicht isolieren. Bei antimonhaltigem Gold. welches ganz dieselben 
Eirscheinungen aufweist, wurde versucht, die $Z$ wischensubstanz herauszulösen. Königswasser zerstörte dieselbe. Auch durch Behandeln des Goldes mit Quecksilber liefs sie sich nicht isolieren. Wohl aber führte die Elektrolyse zum Ziel. Bei Verwendung einer Goldchloridlösung als Elektrolyt überzog sich das antimonhaltige Gold $(2 \% \mathrm{Sb})$ mit einer grauen Haut, die von Zeit zu Zeit abfiel. In derselben liefsen sich noch viele Groldteilchen erkennen, die sich aber nicht entfernen liefsen. Sie enthielt $10 \%$ Antimon. Bei der Elektrolyse in einer Cyangoldlösung zeigten sich in dem Gold Anhäufungen grauer Körperchen, die sich, nachdem etwas mehr Gold gelöst war, abbürsten liefsen. Ihre genaue Zusammensetzung lief's sich nicht feststellen, da eine Probe $22 \%$, eine andere $41 \% \mathrm{Au}$ ergab. Jedenfalls war der Goldgehalt höher, vielleicht ebenfalls der Formel $\mathrm{Au}_{3} \mathrm{Sb}$ entsprechend, denn es ist kaum anzunelmen, dals

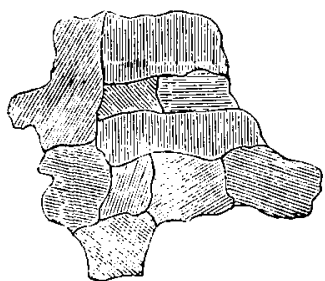

Fig. 22.

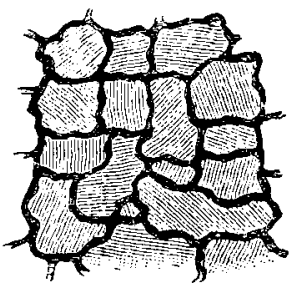

Fig. 23.

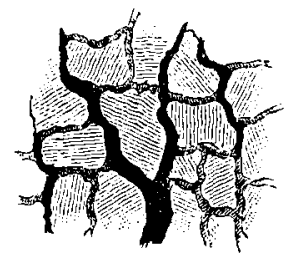

Fig. ist.

die Verbindung sich aus Gold ärmer ausscheirlen sollte, als aus Antimon. Aus einer goldreichen Verbindung wird aber die Elektrolyse leicht ziemlich viel Gold auslösen können.

Bei Wismut und Antimon wurde insofern ein Eintuls eines Gehaltes an Platin, Palladium und Gold auf das Gefüge dieser Metalle beobachtet, als ein solcher Gehalt eine auffallend kleinere Krystallbildung des Wismut und Antimons zur Folge hat. Die sich ausscheidenden Körperchen hindern eben auch hier den festen Zusammenhalt der einzelnen Krystallgruppen.

Der experimentelle Teil vorliegender Arbeit wurde teils im ersten chemischen Universitätslaboratorium zu Berlin, teils im Betriebslaboratorium der Scheideanstalt zu Frankfurt a. M. im Wintersemester 1893/94 und Sommersemester 1894 ausgeführt.

Bei der Redaktion eingegangen am 13. Februar 1895. 